\title{
Derived Categories for Functional Analysis
}

\author{
By
}

\author{
Fabienne Prosmans ${ }^{*}$
}

\begin{abstract}
In this paper, we study the homological algebra of the category $\mathcal{T} c$ of locally convex topological vector spaces from the point of view of derived categories. We start by showing that $\mathcal{T} c$ is a quasi-abelian category in which products and direct sums are exact. This allows us to derive projective and inductive limit functors and to clarify their homological properties. In particular, we obtain strictness and acyclicity criteria. Next, we establish that the category formed by the separated objects of $\mathcal{J} c$ is quasi-abelian and has the same derived category as $\mathcal{T} c$. Since complete objects of $\mathcal{T} c$ do not form a quasi-abelian category, we are lead to introduce the notion of cohomological completeness and to study the derived completion functor. Our main result in this context is an equivalence between the subcategory of $D(\mathcal{T} c)$ formed by cohomologically complete complexes and the derived category of the category of pro-Banach spaces. We show also that, under suitable assumptions, we can reduce the computation of Ext's in $\mathcal{T}_{c}$ to their computation in Ban by means of derived projective limits. We conclude the paper by studying derived duality functors.
\end{abstract}

\section{Contents}

§ 0. Introduction

$\S 1$. Quasi-Abelian Homological Algebra

§ 1.1. Derivation of Quasi-Abelian Categories

$\S$ 1.2. Derivation of Quasi-Abelian Functors

Communicated by M. Kashiwara, March 15, 1999. Revised November 8, 1999. 1991 Mathematics Subject Classification: 46M20, 46M40, 18G50.

Key words and phrases: Homological methods for functional analysis, derived projective and inductive limits, pro-objects, non-abelian homological algebra, quasi-abelian categories.

* Laboratoire Analyse, Géométrie et Applications (UMR 7539), Université Paris 13, Avenue J.-B. Clément, 93430 Villetaneuse, France. 
§ 2. Homological Algebra for Locally Convex Spaces

$\S$ 2.1. The Category $\tau c$ and its Derived Category

$\S$ 2.2. Derived Limits of Locally Convex Spaces

$\S 3$. Separation Functors

$\S$ 3.1. The Category $\widehat{\mathcal{T}} c$

§ 3.2. Equivalence between $D(\mathcal{T} c)$ and $D(\overline{\mathcal{T}} c)$

§ 3.3. The Functors $Z \mathrm{cl}$ and Sep

$\S 4$. Completion Functors

§ 4.1. The Category $\widehat{\tau} c$

$\S$ 4.2. The Functor $\mathrm{Cpl}$ and Cohomological Completeness

§ 4.3. Equivalence between $D_{c c}^{+}(\mathcal{T} c)$ and $D^{+}(\mathcal{P} r o(\mathcal{B} a n))$

§ 4.4. Equivalence between $D^{+}(\mathcal{F} r)$ and $D^{+}\left(\mathcal{P r o}_{\mathbb{N}}(\mathcal{B} a n)\right)$

$\S 5$. Duality Functors

$\S 5.1$. The Inductive Dual

§ 5.2. Relations with the Strong Dual

\section{§ 0 . Introduction}

Our aim in this paper is to study the category $\mathcal{T} c$ of locally convex topological vector spaces from an homological point of view using derived categories. It is well-known that the category $\mathcal{T} c$ is not abelian. Hence, we may not use the classical techniques of homological algebra. We however prove that $\mathcal{T} c$ is quasi-abelian. This allows us to construct the derived category of $\mathcal{T} c$ as explained in $[8,11]$. In this framework, we study the usual functors of functional analysis such as projective and inductive limit, homomorphism, separation, completion and duality functors. A first study of the homological algebra of these functors was done by Palamodov in [7]. Here, by working in derived categories and using the language of pro-objects, we are able to state these results in a more natural way as well as to clarify their proves. This approach also allows us to generalize some of the results to a non countable situation (see e.g. Theorem 4.3.16). Since any complete space is a projective limit of Banach spaces, it is natural to hope to reduce many homological properties of the category $\tau c$ to the corresponding properties of the category $\mathcal{B}$ an of Banach spaces by means of derived projective limit functors. For this reduction, we need both general properties of derived projective limit functors in quasi-abelian categories (see [9]) and more specific properties for the category of topological abelian groups (see [10]).

To fix our notations and make our text more self-contained, we devote the first section to a review of the results on the homological 
algebra of quasi-abelian categories which are used in the rest of the paper.

In the first part of Section 2, we recall the definition of the category $\mathcal{T} c$ of locally convex topological vector spaces. Using the results obtained in [10] for the category $\mathcal{T A} b$ of topological abelian groups, we show that $\mathcal{T} c$ is quasi-abelian. After recalling the fact that the category $\tau c$ has enough injective objects but not enough projective objects, we end with a criterion for checking that a null-sequence of $\mathcal{T} c$ is costrictly exact. In the second part, thanks to the exactness of products and direct sums in $\mathcal{T} c$, we deduce, from the general results of [9] that projective and inductive limit functors are derivable in $\mathcal{T} c$ and that their derived functors are computable by Roos complexes. Then, using results established in [10], we show that if $X$ is a projective system of $\mathcal{T} c$ indexed by a filtering ordered set, the differential $d^{k}$ of its Roos complex is strict for $k \geq 1$ and that $d^{0}$ is strict if and only if $X$ satisfies the condition SC (i.e. if and only if for any $i \in I$ and any absolutely convex neighborhood of zero $U$ in $X_{i}$, there is $j \geq i$ such that

$$
x_{i, k}\left(X_{k}\right) \subset q_{i}\left(\lim _{i \in I} X_{i}\right)+U
$$

for any $k \geq j$ ). As a corollary, we get that a projective system of $\mathcal{T} c$ indexed by a filtering ordered set is lim-acyclic in $\mathcal{T} c$ if and only if it is

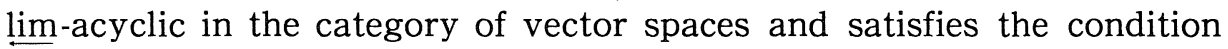
SC. In particular, if the index set $I$ has a cofinal countable subset and if the spaces $X_{i}$ are Fréchet, the condition SC is necessary and sufficient for the lim-acyclicity of the projective system $X$. Note that, in the case of Banach spaces, the condition SC corresponds to the classical topological Mittag-Leffler condition. We conclude by proving that

$$
\mathrm{RHom}_{\mathcal{T} c}(E, F) \simeq \mathrm{R} \underset{q \in Q}{\lim _{p \in P}} \lim _{p \in P} \mathrm{RHom}_{\mathcal{T}_{c}}\left(E_{p}, F_{q}\right)
$$

where $P$ and $Q$ are the (not necessarily countable) systems of semi-norms of $E$ and $F$.

Section 3 is devoted to a cohomological study of the notion of separation. First, we consider the full subcategory $\widetilde{\mathcal{T}} c$ of $\mathcal{T} c$ formed by separated spaces. We prove that $\widetilde{\mathcal{T}} c$ is quasi-abelian and that the left derived functor of the separation functor

$$
\widehat{\text { Sep: }} \mathcal{T} c \rightarrow \widehat{\mathcal{T}} c
$$

is an equivalence of categories. We end by establishing a few properties of the separation and zero closure functors 


$$
\text { Sep }: \mathcal{T} c \rightarrow \mathcal{T} c \quad \text { and } \quad \mathrm{Zcl}: \mathcal{T} c \rightarrow \mathcal{T} c
$$

which will be useful in the next section.

We start Section 4 by considering the category $\widehat{\mathcal{T}} c$ of complete spaces. Since the quotient of a complete space by a closed subspace is not necessarily complete, this category is not quasi-abelian. Hence, we cannot define a derived category of complete locally convex topological vector spaces in a straightforward manner. We show that a way to turn this difficulty is to replace this non-existent derived category by the full subcategory $D_{c c}^{+}(\mathcal{T} c)$ of $D^{+}(\mathcal{T} c)$ formed by cohomologically complete complexes, i.e. the objects $E^{*}$ of $D^{+}\left(\mathcal{T}_{C}\right)$ such that $\operatorname{RCpl}\left(E^{*}\right) \simeq E$, where $\mathrm{Cpl}: \mathcal{T} c \rightarrow \mathcal{T} c$ is the completion functor. Then, we prove an equivalence between the right derived functors of the completion and separation functors. We also give necessary and sufficient conditions, in terms of the derived functor of $\mathrm{Zcl}$, for an object of $\mathcal{T} c$ to be separated, complete or cohomologically complete. Next, we introduce the functor $\mathrm{S}: \mathcal{T} c \rightarrow$ $\operatorname{Pro}(\mathcal{B} a n)$ and $\mathrm{L}: \operatorname{Pro}(\mathcal{B} a n) \rightarrow \mathcal{T} c$ and we relate them by an adjunction formula. After having established that the functor $S$ is exact, we show that the functor $\mathrm{RCpl}: D^{+}(\mathcal{T} c) \rightarrow D^{+}(\mathcal{T} c)$ is canonically isomorphic to $\mathrm{RL}$ o S and we prove that the functors $\mathrm{RL}: D^{+}(\mathcal{P}$ ro $(\mathcal{B} a n)) \rightarrow D_{c c}^{+}(\mathcal{T} c)$ and $\mathrm{S}: D_{c c}^{+}(\mathcal{T} c) \rightarrow D^{+}(\mathcal{P} r o(\mathcal{B} a n))$ are quasi-inverse equivalences of categories. As a corollary, we get a formula reducing the computation of RHom in $\mathcal{T} c$ to that for RHom in $\mathcal{B}$ an by means of derived projective limits. In the last part of this section, after a short study of the quasi-abelian category $\mathcal{F} r$ of Fréchet spaces, we get as a corollary of what has been obtained above that the functor $\mathrm{RL}: D^{+}\left(\mathcal{P r o}_{\mathbb{N}}(\mathcal{B} a n)\right) \rightarrow$ $D^{+}(\mathcal{F} r)$ is an equivalence of categories.

Section 5 is devoted to the study of duality functors. First, we recall some of the properties of the standard duality functor for Banach spaces $\mathrm{D}: \mathcal{B}$ an $\rightarrow(\mathcal{B} a n)^{\text {op }}$. Next, we introduce the inductive dual functor $D_{i}:$ $\mathcal{T} c \rightarrow \mathcal{T} c^{\mathrm{op}}$. We prove that $\mathrm{D}_{\mathrm{i}}$ is left exact and we study its right derived

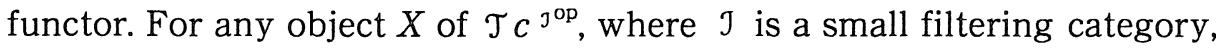
we obtain the formula

$$
\mathrm{RD}_{\mathrm{i}}\left(\mathrm{R} \lim _{i \in \mathrm{J}} X(i)\right) \simeq \mathrm{L} \underset{i \in \mathfrak{J}}{\lim _{i \in \mathrm{J}}}\left(\mathrm{RD}_{\mathrm{i}}(X)\right)(i)
$$

and we show that it is possible to compute $\mathrm{RD}_{\mathrm{i}}$ by means of the exact functor

$$
\operatorname{Pro}(\mathrm{D}): \operatorname{Pro}(\mathcal{B} a n) \rightarrow(\mathcal{I} n d(\mathcal{B} a n))^{\mathrm{op}}
$$

As a corollary, we get that $\mathrm{RD}_{\mathrm{i}}(X) \simeq \mathrm{RD}_{\mathrm{i}}(\mathrm{RCpl}(X)) \simeq \mathrm{RD}_{\mathrm{i}}(\hat{X})$. Finally, 
we show that $R D_{i}$ is canonically isomorphic to the right derived functor of the strong dual functor.

To conclude this introduction, it is a pleasure to thank J.P. Schneiders for the helpful discussions we had during the preparation of this paper.

\section{§ 1. Quasi-Abelian Homological Algebra}

To help the reader and to fix our notations, we recall as in [10] a few basic facts concerning the homological algebra of quasi-abelian categories. We refer to [11] for more details (see also [8]).

\section{§1. 1. Derivation of Quasi-Abelian Categories}

Definition 1.1.1. Let $\mathcal{A}$ be an additive category with kernels and cokernels and let $f: A \rightarrow B$ be a morphism of $\mathcal{A}$. Recall that ker $f$ (resp. coker $f$, $\operatorname{im} f$, coim $f$ ) denotes the kernel (resp. the cokernel, the image, the coimage) of $f$.

We say that $f$ is strict if the canonical morphism

is an isomorphism.

$$
\operatorname{coim} f \rightarrow \operatorname{im} f
$$

Definition 1.1.2. A category $\mathcal{E}$ is quasi-abelian if it is an additive category with kernels and cokernels and

(i) if in a cartesian square

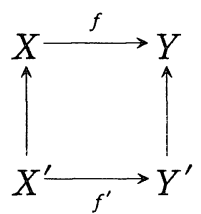

$f$ is a strict epimorphism, then $f^{\prime}$ is a strict epimorphism, 
(ii) if in a cocartesian square

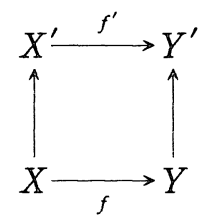

$f$ is a strict monomorphism, then $f^{\prime}$ is a strict monomorphism.

In the rest of the section, $\mathcal{E}$ will denote a quasi-abelian category.

Recall that $C(\mathcal{E})$ is the category of complexes of $\mathcal{E}$ and that $K(\mathcal{E})$ is the category whose objects are the objects of $C(\varepsilon)$ and whose morphisms are the morphisms of $C(\varepsilon)$ modulo homotopy. It is well-known that $K(\varepsilon)$ is a triangulated category.

Definition 1.1.3. (i) A sequence

$$
E \stackrel{f}{\rightarrow} F \stackrel{g}{\rightarrow} G
$$

of $\mathcal{E}$ such that $g \circ f=0$ is strictly exact if $f$ is strict and if the canonical morphism im $f \rightarrow \operatorname{ker} g$ is an isomorphism.

(ii) A complex $X^{\circ}$ of $\mathcal{E}$ is strictly exact in degree $k$ if the sequence

$$
X^{k-1} \stackrel{d^{k-1}}{\longrightarrow} X^{k} \stackrel{d^{k}}{\longrightarrow} X^{k+1}
$$

is strictly exact.

(iii) A complex of $\mathcal{E}$ is strictly exact if it is strictly exact in every degree.

Proposition 1.1.4. The full subcategory $N(\varepsilon)$ of $K(\varepsilon)$ formed by the strictly exact complexes of $\mathcal{E}$ is a null system.

Definition 1.1.5. We define the derived category $D(\mathcal{E})$ of $\mathcal{E}$ as the localization

$$
K(\mathcal{E}) / N(\varepsilon)
$$

of $K(\mathcal{E})$ by $N(\varepsilon)$.

A morphism of $K(\varepsilon)$ which has a strictly exact mapping cone is called a strict quasi-isomorphism. 
Definition 1.1.6. We denote by $D^{\leq 0}(\varepsilon)$ (resp. $D^{\geq 0}(\varepsilon)$ ) the full subcategory of $D(\varepsilon)$ formed by the complexes which are strictly exact in each strictly positive (resp. strictly negative) degree.

Proposition 1.1.7. The pair $\left(D^{\leq 0}(\varepsilon), D^{\geq 0}(\varepsilon)\right)$ is a t-structure on $D(\varepsilon)$. We call it the left $t$-structure of $D(\varepsilon)$.

Definition 1.1.8. We denote $\mathcal{L} \mathcal{H}(\mathcal{E})$ the heart of the left t-structure

$$
\left(D^{\leq 0}(\varepsilon), D^{\geq 0}(\varepsilon)\right) .
$$

We call it the left heart of $D(\varepsilon)$ and we denote

$$
L H^{k}: D(\varepsilon) \rightarrow \mathcal{L} \mathcal{H}(\varepsilon)
$$

the associated cohomological functors.

Proposition 1.1.9. The functor

$$
I: \mathcal{E} \rightarrow \mathcal{L} \mathcal{H}(\varepsilon)
$$

which associates to any object $E$ of $\mathcal{E}$ the complex

$$
0 \rightarrow E \rightarrow 0
$$

where $E$ is in degree 0 is fully faithful.

Remark 1.1.10. Lex $X^{\cdot}$ be an object of $\mathcal{L} \mathcal{H}(\varepsilon)$. By an abuse of notations, we will write

$$
X \cdot \in \mathcal{E}
$$

if $X^{*}$ is isomorphic to $I(E)$ for some object $E$ of $\mathcal{E}$.

Proposition 1.1.11. Let $X^{\prime}$ be an object of $D(\varepsilon)$. Then,

(i) $L H^{k}\left(X^{*}\right)=0$ if and only if $X^{*}$ is strictly exact in degree $k$,

(ii) $L H^{k}\left(X^{*}\right) \in \mathcal{E}$ if and only if $d_{X}^{k-1}$ is strict.

Remark 1.1.12. If we replace the notion of strictly exact sequence by the dual notion of costrictly exact sequence, we obtain a second 
t-structure on $D(\mathcal{E})$. We call it the right $t$-structure of $D(\mathcal{E})$. We denote by $\mathcal{R} \mathcal{H}(\mathcal{E})$ its heart and by $R H^{n}$ the corresponding cohomological functors.

\section{$\S 1.2$. Derivation of Quasi-Abelian Functors}

Let $F: \mathcal{E} \rightarrow \mathcal{F}$ be an additive functor between quasi-abelian categories.

Definition 1.2.1. The functor $F$ is left exact (resp. exact) if it transforms any strictly exact sequence

$$
0 \rightarrow E^{\prime} \rightarrow E \rightarrow E^{\prime \prime} \rightarrow 0
$$

of $\varepsilon$ into the strictly exact sequence

$$
0 \rightarrow F\left(E^{\prime}\right) \rightarrow F(E) \rightarrow F\left(E^{\prime \prime}\right) \quad\left(\text { resp. } 0 \rightarrow F\left(E^{\prime}\right) \rightarrow F(E) \rightarrow F\left(E^{\prime \prime}\right) \rightarrow 0\right)
$$

Definition 1.2.2. Denote as usual

$$
\mathrm{Q}_{\mathcal{E}}: K^{+}(\mathcal{E}) \rightarrow D^{+}(\mathcal{E}), \quad \mathrm{Q}_{\mathcal{F}}: K^{+}(\mathcal{F}) \rightarrow D^{+}(\mathcal{F})
$$

the canonical functors. Assume we are given a triangulated functor

$$
G: D^{+}(\mathcal{E}) \rightarrow D^{+}(\mathcal{F})
$$

and a morphism

$$
g: Q_{\mathcal{F}} \circ K^{+}(F) \rightarrow G \circ Q_{\mathcal{E}}
$$

Then, $(G, g)$ is a right derived functor of $F$ if for any other such pair $\left(G^{\prime}, g^{\prime}\right)$, there is a unique morphism

$$
h: G \rightarrow G^{\prime}
$$

making the diagram 


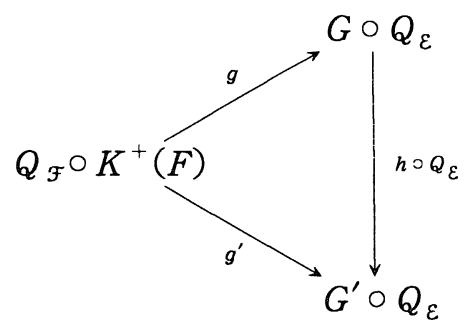

commutative. The functor $F$ is right derivable if it has a right derived functor. One denotes $R F$ a canonical choice of a derived functor of $F$.

Definition 1.2.3. Let $F$ be a right derivable functor. An object $I$ of $\mathcal{E}$ is called $F$-acyclic if $F(I) \simeq R F(I)$.

Definition 1.2.4. A full subcategory $\mathcal{J}$ of $\mathcal{E}$ is $F$-injective if

(i) for any object $E$ of $\mathcal{E}$, there is an object $I$ of $\mathcal{J}$ and a strict monomorphism $E \rightarrow I$,

(ii) in any strictly exact sequence $0 \rightarrow E^{\prime} \rightarrow E \rightarrow E^{\prime \prime} \rightarrow 0$ of $\mathcal{E}$ where $E^{\prime}$ and $E$ are objects of $\mathcal{J}$, then $E^{\prime \prime}$ is an object of $\mathcal{J}$ and the sequence

$$
0 \rightarrow F\left(E^{\prime}\right) \rightarrow F(E) \rightarrow F\left(E^{\prime \prime}\right) \rightarrow 0
$$

is strictly exact in $\mathcal{F}$.

Proposition 1.2.5. If $\mathcal{J}$ is an F-injective subcategory of $\mathcal{E}$, then for any object $X^{\cdot}$ of $C^{+}(\mathcal{E})$, there is a strict quasi-isomorphism

$$
u^{\cdot}: X^{\cdot} \rightarrow I^{\cdot}
$$

such that, for any $k, I^{k}$ is an object of $\mathcal{J}$ and $u^{k}: X^{k} \rightarrow I^{k}$ is a strict monomorphism. (In such a case, we call $I^{*}$ an F-injective resolution of $X^{*}$.)

Proposition 1.2.6. Assume $\mathcal{E}$ has an F-injective subcategory $\mathcal{J}$. Then, the functor $F: \mathcal{E} \rightarrow \mathcal{F}$ has a right derived functor

$$
R F: D^{+}(\mathcal{E}) \rightarrow D^{+}(\mathcal{F}),
$$

and for any $F$-injective resolution $I^{\prime}$ of $X^{\prime}$ we have a canonical isomorphism

$$
R F\left(X^{\cdot}\right) \simeq F\left(I^{\cdot}\right)
$$


in $D^{+}(\mathcal{F})$. In particular, the objects of $\mathrm{J}$ are F-acyclic.

Definition 1.2.7。

(i) An object $I$ of $\mathcal{E}$ is called injective if the functor

is exact.

$$
\operatorname{Hom}_{\mathcal{E}}\left({ }^{\circ}, I\right): \mathcal{E}^{\mathrm{op}} \rightarrow \mathcal{A} b
$$

(ii) The category $\mathcal{E}$ has enough injective objects if for any object $E$ of $\mathcal{E}$, there is a strict monomorphism $E \rightarrow I$ where $I$ is an injective object of $\varepsilon$.

Proposition 1.2.8. If $\mathcal{E}$ has enough injective objects, then the full subcategory $\mathcal{J}$ of $\mathcal{E}$ formed by injective objects is an F-injective subcategory for any additive functor $F: \mathcal{E} \rightarrow \mathcal{F}$. In particular, any such functor $F$ is right derivable and any object $X^{\cdot}$ of $C^{+}(\mathcal{E})$ has a resolution $I^{\cdot}$ with injective components.

Remark 1.2.9. Although we will not state them explicitly here, we have of course dual results for left derivable functors. As usual, in the dual vocabulary, the word "injective" is replaced by the word "projective".

\section{§ 2. Homological Algebra for Locally Convex Spaces}

\section{§2.1. The Category $\mathcal{T} c$ and its Derived Category}

Recall that a topological $\mathbb{C}$-vector space $E$ is a $\mathbb{C}$-vector space endowed with a topology having the property that both the scalar multiplication

$$
\circ: \mathbb{C} \times E \rightarrow E
$$

and the addition

$$
+: E \times E \rightarrow E
$$

are continuous. A topological $\mathbb{C}$-vector space is locally convex if 0 has a basis of absolutely convex neighborhoods. It is well known that the topology of a locally convex vector space is always given by a system of 
semi-norms (i.e. a set $P$ of semi-norms on $E$ such that for any $p, p^{\prime}$ in $P$ there is $p^{\prime \prime}$ such that $\left.p^{\prime \prime} \geq \sup \left\{p, p^{\prime}\right\}\right)$. This system of semi-norms may be chosen to be the set of gauge semi-norms associated to a basis of absolutely convex neighborhoods of 0 . Conversely, if $P$ is a system of semi-norms on $E$, then the set $\left\{b_{p}(r): p \in P, r>0\right\}$ where $b_{p}(r)=\{e \in$ $E: p(e)<r\}$ forms a basis of absolutely convex neighborhoods of 0 on $E$.

Definition 2.1.1. We denote by $\mathcal{T} c$ the category whose objects are the locally convex topological vector spaces and whose morphisms are the continuous linear maps between locally convex topological vector spaces.

Definition 2.1.2. (i) Let $E$ be an object of $\mathcal{T} c$ and let $H$ be a subspace of $E$. The locally convex topology on $H$ associated to the system of semi-norms $\left\{p_{i H}: p \in P\right\}$ where $P$ is a system of semi-norms of $E$ is called the induced topology. If $V$ is a basis of absolutely convex neighborhoods of 0 on $E$, then the set $\{V \cap H: V \in \mathcal{V}\}$ forms a basis of absolutely convex neighborhoods of 0 on $H$.

(ii) Let $E$ be an object of $\mathcal{T} c$ and let $H$ be a subspace of $E$. For any semi-norm $p$ of $E$, we denote by $p$ the semi-norm of $E / H$ defined by

$$
p(x)=\inf _{e \in q^{-1}(x)} p(e)
$$

where $q: E \rightarrow E / H$ is the canonical epimorphism. The locally convex topology on $E / H$ associated to the system of semi-norms $\{p: p \in P\}$ where $P$ is a system of semi-norms of $E$ is called the quotient topology. If $V$ is a basis of absolutely convex neighborhoods of 0 on $E$, then the set $\{q(V): V \in \mathcal{V}\}$ defines a basis of absolutely convex neighborhoods of 0 on $E / H$.

Proposition 2.1.3. Any family $\left\{E_{\alpha}\right\}_{\alpha \in A}$ of objects of $\mathcal{T} c$ has a product. This product is obtained by endowing the $\mathbb{C}$-vector space

$$
\prod_{\alpha \in A} E_{\alpha}=\left\{\left(e_{\alpha}\right)_{\alpha \in A}: e_{\alpha} \in E_{\alpha}\right\}
$$

with the locally convex topology associated to the family of semi-norms

$$
\left\{\sup \left(p_{1} \circ \pi_{\alpha_{1}}, \ldots, p_{N} \circ \pi_{\alpha_{N}}\right): \alpha_{1}, \ldots, \alpha_{N} \in A, p_{1} \in P_{\alpha_{1}}, \ldots, p_{N} \in P_{\alpha_{N}}\right\}
$$

where $\pi_{\alpha}: \prod_{\alpha \in A} E_{\alpha} \rightarrow E_{\alpha}$ is the canonical projection and $P_{\alpha}$ is a system of semi-norms of $E_{\alpha}$. A basis of absolutely convex neighborhoods of 0 in 
$\Pi_{\alpha \in A} E_{\alpha}$ is given by the subsets of the form $\Pi_{\alpha \in A} W_{\alpha}$, where each $W_{\alpha}$ is an absolutely convex neighborhood of zero in $E_{\alpha}$, the set $\left\{\alpha: W_{\alpha} \neq E_{\alpha}\right\}$ being finite.

Remark 2.1.4. Hereafter, $\langle A\rangle$ denotes as usual the absolutely convex hull of a subset $A$ of a $\mathbb{C}$-vector space $E$.

Proposition 2.1.5. Any family $\left\{E_{\alpha}\right\}_{\alpha \in A}$ of objects of $\mathcal{T}_{c}$ has a direct sum. This direct sum is obtained by endowing the $\mathbb{C}$-vector space

$$
\bigoplus_{\alpha \in A} E_{\alpha}=\left\{\left(e_{\alpha}\right)_{\alpha \in A}: e_{\alpha} \in E_{\alpha}, e_{\alpha} \neq 0 \text { for finitely many } \alpha\right\}
$$

with the locally convex topology associated to the family of semi-norms

$$
\left\{\sum_{\alpha \in A} c_{\alpha} p_{\alpha} \circ \pi_{\alpha}: c_{\alpha}>0, p_{\alpha} \in P_{\alpha}\right\}
$$

where $\pi_{\alpha}: \oplus_{\alpha \in A} E_{\alpha} \rightarrow E_{\alpha}$ is the canonical projection and $P_{\alpha}$ is a system of semi-norms on $E_{\alpha}$. A basis of absolutely convex neighborhoods of 0 in $\oplus_{\alpha \in A} E_{\alpha}$ is given by the subsets of the form $\left\langle\cup_{\alpha \in A} \sigma_{\alpha}\left(W_{\alpha}\right)\right\rangle$ where each $W_{\alpha}$ is an absolutely convex neighborhood of zero in $E_{\alpha}$ and $\sigma_{\alpha}: E_{\alpha} \rightarrow \bigoplus_{\alpha \in A} E_{\alpha}$ is the canonical embedding.

Proposition 2.1.6. Let $\left(X_{i}\right)_{i \in I}$ be a family of $\mathcal{T} c$. For any normed space $X$, we have

$$
\operatorname{Hom}_{\mathcal{T}_{c}}\left(\prod_{i \in I} X_{i}, X\right) \simeq \bigoplus_{i \in I} \operatorname{Hom}_{\mathcal{T}_{c}}\left(X_{i}, X\right)
$$

Proof. Work e.g. as in [6, Chap. IV, § 22, 5.(2) (p. 284)].

Remark 2.1.7. Note that the preceding result does not hold if the norm of $X$ is replaced by a semi-norm.

Proposition 2.1.8. The category $\mathcal{T} c$ is an additive category with kernels and cokernels. More precisely, if $f: E \rightarrow F$ is a morphism of $\mathcal{T} c$, then:

(i) the subspace $f^{-1}(0)$ of $E$ endowed with the induced topology together with the canonical monomorphism $f^{-1}(0) \rightarrow E$ form a kernel of $f$;

(ii) the quotient space $F / f(E)$ endowed with the quotient topology together with the canonical epimorphism $q: F \rightarrow F / f(E)$ form a cokernel of 
$f$;

(iii) the image of $f$ is the subspace $f(E)$ of $F$ endowed with the induced topology;

(iv) the coimage of $f$ is the quotient space $E / f^{-1}(0)$ endowed with the quotient topology.

Corollary 2.1.9. Let $f: E \rightarrow F$ be a morphism of $\mathcal{T} c$. The following conditions are equivalent:

(i) fis strict,

(ii) $f$ is relatively open (i.e. for any neighborhood of zero $V$ in $E$, there is a neighborhood of zero $V^{\prime}$ in $F$ such that $f(V) \supset f(E) \cap V^{\prime}$ ),

(iii) for any semi-norm $p$ of $E$, there is a semi-norm $q$ of $F$ and $C>0$ such that

$$
\inf _{e \in \operatorname{ker} f} p(x+e) \leq C q(f(x)) \quad \forall x \in E .
$$

Hereafter, we will use freely the properties of the category $\mathcal{T} \mathcal{A} b$ of topological abelian groups established in [10].

Lemma 2.1.10. Denote by $\psi: \mathcal{T} c \rightarrow \mathcal{T} \mathcal{A} b$ the canonical functor.

(i) The functor $\phi$ is kernel and cokernel preserving.

(ii) A morphism $f: E \rightarrow F$ of $\mathcal{T}_{c}$ is an isomorphism in $\mathcal{T} c$ if and only if $\psi(f)$ is an isomorphism in $\mathcal{T} \mathcal{A} b$.

(iii) A morphism $f: E \rightarrow F$ is strict in $\mathcal{T} c$ if and only if $\phi(f)$ is strict in $\mathcal{T} \mathcal{A} b$.

(iv) A sequence $E \rightarrow F \rightarrow G$ of $\mathcal{T} c$ is strictly exact in $\mathcal{T} c$ if and only if it's image by $\phi$ is strictly exact in $\mathcal{T} \mathcal{A} b$.

Proposition 2.1.11. The category $\mathcal{T} c$ is quasi-abelian.

Proof. We know that $\mathcal{T}_{c}$ is additive and has kernels and cokernels. 
(i) Consider a cartesian square

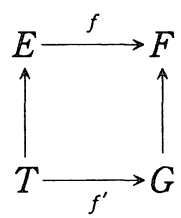

where $f$ is a strict epimorphism. Since this square is cartesian in $\mathcal{T} \mathcal{A} b, f$ is a strict epimorphism in $\mathcal{T} \mathcal{A} b$ and the category $\mathcal{T} \mathcal{A} b$ is quasi-abelian, it follows that $f^{\prime}$ is a strict epimorphism in $\mathcal{T} \mathcal{A} b$. Therefore, $f^{\prime}$ is a strict epimorphism of $\mathcal{T} c$.

(ii) Using the same kind of arguments, in the cocartesian square,

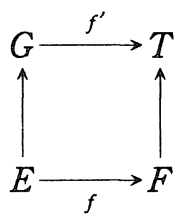

where $f$ is a strict monomorphism, $f^{\prime}$ is also a strict monomorphism.

Proposition 2.1.12. (i) Any vector space E endowed with the weakest locally convex topology is an injective object of $\mathcal{T} c$.

(ii) Let $M$ be an arbitrary non-empty set. The Banach space $l^{\infty}(M)$ of all bounded maps of $M$ into $\mathbb{C}$, with the norm

$$
\|f\|_{l^{\infty}(M)}=\sup \{|f(m)|: m \in M\}
$$

is an injective object of $\mathcal{T} c$.

(iii) For any object $X$ of $\mathcal{T} c$ with $P$ as system of semi-norms, there is a strict monomorphism

$$
X \rightarrow \overline{\{0\}}^{X} \times \prod_{p \in P} l^{\infty}\left(b_{p}(1)^{\circ}\right)
$$

where

$$
\overline{\{0\}}^{X}=\bigcap_{p \in P}\{x \in X: p(x)=0\}
$$

is endowed with the weakest topology and $b_{p}(1)^{\circ}$ denotes the polar of $b_{p}(1)$ in $X^{\prime}$. Hence, the category $\mathcal{T}_{c}$ has enough injective objects.

(iv) The category $\mathcal{T} c$ has not enough projective objects.

Proof. For (i), (ii), (iii), see [7]. For (iv), see[4]. 
Proposition 2.1.13. A sequence $E \rightarrow F \rightarrow G$ is costrictly exact in $\mathcal{T} c$ if and only if

(a) it is algebraically exact;

(b) the sequence

$$
\operatorname{Hom}_{\tau_{c}}\left(G, l^{\infty}(I)\right) \rightarrow \operatorname{Hom}_{\tau_{c}}\left(F, l^{\infty}(I)\right) \rightarrow \operatorname{Hom}_{\tau_{c}}\left(E, l^{\infty}(I)\right)
$$

is exact for any set $I$.

Proof. Applying [11, Proposition 1.3.23] and using Proposition 2.1.12, we know that the sequence $E \rightarrow F \rightarrow G$ is costrictly exact in $\mathcal{T}_{c}$ if and only if the sequence

$$
\operatorname{Hom}_{\tau_{c}}(G, J) \rightarrow \operatorname{Hom}_{\tau_{c}}(F, J) \rightarrow \operatorname{Hom}_{\tau_{c}}(E, J)
$$

is exact for any injective object $J$ of $\mathcal{T} c$ of the form

$$
V \times \prod_{k \in K} l^{\infty}\left(I_{k}\right)
$$

where $V$ is endowed with the weakest topology. Since

$$
\operatorname{Hom}_{\tau_{c}}\left(X, \prod_{i \in I} Y_{i}\right) \simeq \prod_{i \in I} \operatorname{Hom}_{\tau_{c}}\left(X, Y_{i}\right)
$$

we see that $E \rightarrow F \rightarrow G$ is costrictly exact if and only if the sequences

$$
\operatorname{Hom}_{\tau_{c}}\left(G, l^{\infty}(I)\right) \rightarrow \operatorname{Hom}_{\mathcal{I}_{c}}\left(F, l^{\infty}(I)\right) \rightarrow \operatorname{Hom}_{\mathcal{T}_{c}}\left(E, l^{\infty}(I)\right)
$$

and

$$
\operatorname{Hom}_{\tau_{c}}(G, V) \rightarrow \operatorname{Hom}_{\tau_{c}}(F, V) \rightarrow \operatorname{Hom}_{\tau_{c}}(E, V)
$$

are exact for any set $I$ and any vector space $V$. Denote $V$ the category of $\mathbb{C}$-vector spaces. Since any object of $\mathcal{V}$ is injective and since

$$
\operatorname{Hom}_{\tau_{c}}(X, V)=\operatorname{Hom}_{v}(X, V),
$$

the conclusion follows easily. 


\section{§ 2.2. Derived Limits of Locally Convex Spaces}

Proposition 2.2.1. The category $\mathcal{T} c$ is complete and cocomplete. More precisely, let $F: \mathcal{J} \rightarrow \mathcal{T} c$ be a functor from a small category $\mathcal{J}$ to the category $\mathcal{T}$ c. Denote by $\operatorname{Ar}(\mathcal{J})$ the set of morphisms of $\mathcal{J}$, and, for any morphism $\alpha \in \operatorname{Ar}(\mathcal{J})$, denote $\operatorname{org}(\alpha)$ (resp. $\operatorname{ext}(\alpha)$ ) the origin (resp. the extremity) of $\alpha$.

(i) Consider the morphism

$$
f: \prod_{i \in \mathrm{Ob}(\mathcal{J})} F(i) \rightarrow \prod_{\alpha \in \operatorname{Ar}(\mathcal{J})} F(\operatorname{ext}(\alpha))
$$

defined by setting

$$
\pi_{\alpha} \circ f=\pi_{\operatorname{ext}(\alpha)}-F(\alpha) \circ \pi_{\mathrm{org}(\alpha)} \quad \forall \alpha \in \operatorname{Ar}(\mathcal{J})
$$

where

$$
\pi_{\alpha}: \prod_{\alpha \in \operatorname{Ar}(\mathcal{\jmath})} F(\operatorname{ext}(\alpha)) \rightarrow F(\operatorname{ext}(\alpha)) \quad \text { and } \quad \pi_{i}: \prod_{i \in \mathrm{Ob}(\mathcal{\jmath})} F(i) \rightarrow F(i)
$$

are the canonical projections. Denote

$$
x: \operatorname{ker} f \rightarrow \prod_{i \in \mathrm{Ob}(\mathcal{J})} F(i)
$$

the canonical monomorphism. Then, ker $f$ together with the morphisms

$$
q_{i}=\pi_{i} \circ x: \operatorname{ker} f \rightarrow F(i)
$$

form a projective limit of $F$ in $\mathcal{T}$ c. We denote it by $\lim _{i \in J} F(i)$.

(ii) Consider the morphism

$$
f: \bigoplus_{\alpha \in \operatorname{Ar}(\jmath)} F(\operatorname{org}(\alpha)) \rightarrow \bigoplus_{i \in \mathrm{Ob}(\jmath)} F(i)
$$

defined by setting

$$
f \circ \sigma_{\alpha}=\sigma_{\mathrm{org}(\alpha)}-\sigma_{\mathrm{ext}(\alpha)} \circ F(\alpha) \quad \forall \alpha \in \operatorname{Ar}(\mathcal{J})
$$

where

$$
\sigma_{\alpha}: F(\operatorname{org}(\alpha)) \rightarrow \bigoplus_{\alpha \in \operatorname{Ar}(\mathcal{J})} F(\operatorname{org}(\alpha)) \quad \text { and } \quad \sigma_{i}: F(i) \rightarrow \bigoplus_{i \in \mathrm{Ob}(\mathcal{\jmath})} F(i)
$$


are the canonical embeddings. Denote

$$
x: \bigoplus_{i \in \mathrm{Ob}(\jmath)} F(i) \rightarrow \operatorname{coker} f
$$

the canonical morphism. Then, coker $f$ together with the morphisms

$$
r_{i}=x \circ \sigma_{i}: F(i) \rightarrow \operatorname{coker} f
$$

form an inductive limit of $F$ in $\mathcal{T}$ c. We denote it by $\underset{i \in \mathfrak{\jmath}}{\lim _{i \in}} F(i)$.

Proposition 2.2.2. Products and direct sums are exact in $\mathcal{T}$ c. In particular, for any small category $\mathcal{J}$, the functor

$$
\lim _{i \in \mathfrak{J}}: \mathcal{T} c^{\mathcal{J}^{\mathrm{op}}} \rightarrow \mathcal{T} c
$$

is right derivable and for any object $X$ of $\mathcal{T}_{c}{ }^{\mathrm{op}}$, we have

$$
\mathrm{R} \lim _{i \in \mathcal{J}} X(i) \simeq R^{\cdot}(\mathcal{J}, X)
$$

where $R^{\cdot}(\mathcal{J}, X)$ is the positive Roos complex associated to $X$ in [9]. Similarly, for any small category $\mathcal{J}$, the functor

$$
\lim _{i \in \mathfrak{J}}: \mathcal{T} c^{\mathcal{J}} \rightarrow \mathcal{T} c
$$

is left derivable and for any object $X$ of $\mathcal{T} c^{\mathcal{J}}$, we have

$$
\mathrm{L} \lim _{i \in \mathcal{J}} X(i) \simeq R .(\mathcal{J}, X)
$$

where $R .(\mathcal{J}, X)$ is the negative Roos complex associated to $X$ in [9].

Proof. The exactness of products and direct sums follows at once from Propositions 2.1.3 and 2.1.5. The existence and structure of derived limits is then a consequence of [9, Propositions 3.3.3 and 3.3.4].

Lemma 2.2.3. Let $\mathcal{J}$ be a small category. For any Banach space $B$ and any object $X$ of $\mathcal{T}_{c}{ }^{{ }^{\mathrm{op}}}$, we have the isomorphism

$$
\operatorname{Hom}\left(R^{\cdot}(\mathcal{J}, X), B\right) \simeq R .\left(\mathcal{J}^{\mathrm{op}}, \operatorname{Hom}(X, B)\right)
$$

Proof. For any Banach space $B$ and any $k \geq 0$, we have 


$$
\begin{aligned}
\left(\operatorname{Hom}\left(R^{\cdot}(\mathcal{J}, X), B\right)\right)^{-k} & \simeq \prod_{p \in \mathbb{Z}} \operatorname{Hom}_{\mathcal{T} c}\left(R^{p}(\mathcal{J}, X), B^{p-k}\right) \\
& \simeq \operatorname{Hom}_{\mathcal{T} c}\left(R^{k}(\mathcal{J}, X), B\right) .
\end{aligned}
$$

So,

$$
\begin{aligned}
\left(\operatorname{Hom}\left(R^{\cdot}(\mathcal{J}, X), B\right)\right)^{-k} & \simeq \operatorname{Hom}_{\mathcal{J} c}\left(\prod_{i_{0} \stackrel{\alpha_{1}}{\longrightarrow} \cdots \stackrel{\alpha_{k}}{\longrightarrow} i_{k}} X\left(i_{0}\right), B\right) \\
& \simeq \bigoplus_{i_{0} \stackrel{\alpha_{1}}{\longrightarrow} \cdots \stackrel{\alpha_{k}}{\longrightarrow} i_{k}} \operatorname{Hom}(X, B)\left(i_{0}\right) \\
& \simeq R_{k}\left(\mathcal{J}^{\mathrm{op}}, \operatorname{Hom}(X, B)\right) \\
& \simeq\left(R \cdot\left(\mathcal{J}^{\mathrm{op}}, \operatorname{Hom}(X, B)\right)\right)^{-k}
\end{aligned}
$$

where the second isomorphism follows from Proposition 2.1.6. The conclusion follows.

Proposition 2.2.4. Let $\mathcal{J}$ be a small filtering category. Consider a Banach space $B$ and an object $X$ of $\mathcal{T}_{c}{ }^{\mathcal{J}^{\text {op }}}$ such that $R \lim _{i \in \mathcal{J}} X(i) \in D^{b}(\mathcal{T} c)$. Then, we have the isomorphism

$$
\mathrm{RHom}\left(\mathrm{R} \lim _{i \in \mathrm{J}} X(i), B\right) \simeq \lim _{i \in \mathrm{J}}(\operatorname{RHom}(X, B))(i) .
$$

Proof. We know that $\mathrm{R} \underset{i \in \mathcal{J}}{\lim } X(i) \simeq R^{\prime}(\mathcal{J}, X)$. By "dévissage", it is sufficient to prove the result when $B$ is an injective Banach space. In this case, we have successively

$$
\begin{aligned}
& \mathrm{RHom}\left(\mathrm{R} \lim _{i \in \mathcal{J}} X(i), B\right) \simeq \operatorname{Hom}\left(R^{\cdot}(\mathcal{J}, X), B\right) \\
& \simeq R .\left(\mathcal{J}^{\mathrm{op}}, \operatorname{Hom}(X, B)\right) \\
& \simeq \mathrm{L} \underset{i \in \mathfrak{J}}{\lim _{i \in \mathrm{m}}} \operatorname{Hom}(X, B)(i) \\
& \simeq \lim _{i \in \jmath} \operatorname{Hom}(X, B)(i) \\
& \simeq \lim _{i \in \mathcal{J}}(\operatorname{RHom}(X, B))(i)
\end{aligned}
$$


where the isomorphism $\left({ }^{*}\right)$ follows from Lemma 2.2.3. The isomorphism $\left.{ }^{(* *}\right)$ follows from the fact that the functor $\lim _{i \in \mathfrak{J}}: \mathcal{A} b^{\mathcal{J}} \rightarrow \mathcal{A} b$ is exact.

Definition 2.2.5. Let $\mathcal{J}$ be a small filtering category. By a result of Deligne(see [1, Proposition 8.1.6]), there is a cofinal functor

$$
\Phi: I \rightarrow \mathcal{J}
$$

where $I$ is a small filtering ordered set. Since any non empty set of cardinal numbers has a minimum, we may assume that $I$ has the smallest possible cardinality. This cardinality will be called the cofinality of $\mathcal{J}$. We denote it by $\operatorname{cf}(\mathcal{J})$.

Notation 2.2.6. For any $k \in \mathbb{N}$, we denote by $\omega_{k}$ the $(k+1)$-th infinite cardinal number. For example, $\omega_{0}$ is the cardinality of $\mathbb{N}, \omega_{1}$ is the smallest cardinal number which is strictly greater than $\omega_{0}$, and so on.

Proposition 2.2.7. Assume $\mathcal{J}$ is a small filtering category such that $\operatorname{cf}(\mathcal{J})<\omega_{k}$ for some $k<\omega_{0}$. Then, for any functor $X: \mathcal{J}^{\mathrm{op}} \rightarrow \mathcal{T} c$ we have

$$
L H^{n}\left(\mathrm{R} \lim _{i \in \mathfrak{\jmath}} E(i)\right)=0 \quad \forall n \geq k+1 .
$$

Similarly, for any functor

$$
X: \mathcal{J} \rightarrow \mathcal{T}_{c}
$$

we have

$$
R H^{n}\left(\mathrm{~L}_{i \in \mathcal{J}} X(i)\right)=0 \quad \forall n \geq k+1 .
$$

Proof. This follows from [9, Theorem 5.2.4].

Definition 2.2.8. Let $I$ be a filtering ordered set. We say that a projective system $X$ of $\tau c^{I^{\text {op }}}$ satisfies condition SC if for any $i \in I$ and any absolutely convex neighborhood $U$ of zero in $X_{i}$, there is $j \geq i$ such that

$$
x_{i, k}\left(X_{k}\right) \subset q_{i}\left(\varliminf_{i \in I} X_{i}\right)+U \quad \forall k \geq j .
$$


Proposition 2.2.9. Let I be a filtering ordered set and let $X$ be an object of $\mathcal{T} c^{I^{\mathrm{op}}}$. Then:

(a) we have

$$
L H^{1}\left(\mathrm{R} \lim _{i \in I} X_{i}\right) \in \mathcal{T} c
$$

if and only if $X$ satisfies condition $S C$.

In particular, the differential $d_{R^{\prime}(I, X)}^{0}$ of the Roos complex of $X$ is strict if and only if $X$ satisfies condition $S C$.

(b) we have

$$
L H^{k}\left(\mathrm{R} \varliminf_{i \in I} X_{i}\right) \in \mathcal{T}_{c} \quad \forall k \geq 2 .
$$

In particular, the differential $d_{R^{(}(I, X)}^{k}$ of the Roos complex of $X$ is strict for $k$ $\geq 1$.

Proof. This follows directly from [10, Theorems 4.3 and 4.4] and from Lemma 2.1.10.

Corollary 2.2.10. Let $\Phi: \mathcal{T} c \rightarrow \nu$ be the functor which associates to any object $X$ of $\mathcal{T} c$, the vector space $X$. Let I be a filtering ordered set. If $X$ is an object of $\mathcal{T} c^{I^{\mathrm{op}}}$, then the following conditions are equivalent:

(i) $\lim _{i \in I} X_{i} \simeq \mathrm{R} \lim _{i \in I} X_{i}$,

(ii) $\lim _{i \in I} \Phi\left(X_{i}\right) \simeq \mathrm{R} \lim _{i \in I} \Phi\left(X_{i}\right)$ and $X$ satisfies condition $S C$.

Proof. This follows from [10, Corollary 4.5].

Proposition 2.2.11. Let I be a filtering ordered set with a countable cofinal subset. Let $X$ be an object of $\mathcal{T} c^{{ }^{I D}}$ such that for any $i \in I, X_{i}$ is a Fréchet space. Then, $X$ is $\lim _{i \in I}$-acyclic if and only if for any $i \in I$ and any neighborhood of zero $U$ in $X_{i}$, there is $j \geq i$ such that

$$
x_{i, j}\left(X_{j}\right) \subset U+x_{i, k}\left(X_{k}\right) \quad \forall k \geq j .
$$

Proof. This follows from [10, Theorem 5.6].

Corollary 2.2.12. Let I be a filtering ordered set with a countable 
cofinal subset. Let $E$ be an object of $\mathcal{T} c^{{ }^{0 \mathrm{p}}}$ such that for any $i \in I, E_{i}$ is a Banach space. Then, $E$ is $\lim _{i \in I}$-acyclic if and only if for any $i \in I$, there is $j \geq i$ such that

$$
\overline{e_{i, j}\left(E_{j}\right)}=\overline{e_{i, k}\left(E_{k}\right)} \quad \forall k \geq j
$$

Definition 2.2.13. Let $E$ be an object of $\mathcal{T} c$ with $P$ as system of semi-norms. For any $p \in P$, we denote $E_{p}$ the semi-normed space obtained by endowing $E$ with the semi-norm $p$.

Proposition 2.2.14. Let $E$ be an object of $\mathcal{T} c$ with $P$ as system of semi-norms. We have the isomorphism

$$
E \simeq \mathrm{R} \lim _{p \in P} E_{p}
$$

in $D^{+}(\mathcal{T} c)$.

Proof. Recall that

$$
E \simeq \lim _{p \in P} E_{p} .
$$

Moreover, if we forget the topologies, we have $E=E_{p}$ and in $D^{+}(\mathcal{V})$, we have successively

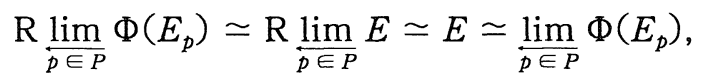

where the second isomorphism follows from [9, Corollary 7.3.7 and Proposition 7.3.9]. Through the isomorphism (*), the canonical morphism $q_{p}: \lim _{p \in \bar{P}} E_{p} \rightarrow E_{p}$ becomes the identity map $E \rightarrow E_{p}$. Hence, it is clear that condition $\mathrm{SC}$ is satisfied and by Corollary 2.2.10, we get $\lim _{p \in P} E_{p}$ $\simeq \mathrm{R} \varliminf_{p \in P} E_{p}$.

Lemma 2.2.15. Let $E$ be an object of $\mathcal{T} c$ with $P$ as system of semi-norms. For any semi-normed space $X$, we have the isomorphism

$$
\operatorname{Hom}_{\mathcal{T}_{c}}(E, X) \simeq \lim _{p \in P} \operatorname{Hom}_{\mathcal{T}_{c}}\left(E_{p}, X\right) .
$$

Proof. We know that for any $f \in \lim _{p \in P} \operatorname{Hom}_{\mathcal{T} c}\left(E_{p}, X\right)$ there is a 
semi-norm $p$ of $P$ and $f_{p} \in \operatorname{Hom}_{\tau_{c}}\left(E_{p}, X\right)$ such that $f=r_{p}\left(f_{p}\right)$. Then, we define the morphism

$$
u: \lim _{p \in P} \operatorname{Hom}_{\tau_{c}}\left(E_{p}, X\right) \rightarrow \operatorname{Hom}_{\mathcal{T}_{c}}(E, X)
$$

by setting $u(f)=f_{p} \circ e_{p}$ where $e_{p}: E \rightarrow E_{p}$ is the identity map. One checks easily that this definition is meaningful and that $u$ is bijective.

Proposition 2.2.16。 Let $E, F$ be objects of $\mathcal{T} c$ with $P$ and $Q$ as systems of semi-norms. We have the canonical isomorphism

$$
\operatorname{RHom}_{\tau_{c}}(E, F) \simeq \mathrm{R} \underset{q \in Q}{\lim _{q \in P}} \lim _{p \in P} \operatorname{RHom}_{\tau_{c}}\left(E_{p}, F_{q}\right) .
$$

Proof. Using Proposition 2.2.14 and [9, Proposition 3.6.3], we get successively

$$
\mathrm{RHom}_{\mathcal{T}_{c}}(E, F) \simeq \mathrm{RHom}_{\mathcal{T}_{c}}\left(E, \mathrm{R} \varliminf_{q \in Q}^{\varliminf_{q}} F_{q}\right) \simeq \mathrm{R} \varliminf_{q \in Q}^{\lim _{q}} \operatorname{RHom}_{\mathcal{T}_{c}}\left(E, F_{q}\right) .
$$

Let $I_{q}$ be a resolution of $F_{q}$ by injective semi-normed spaces. We have

$$
\operatorname{RHom}_{\mathcal{T}_{c}}\left(E, F_{q}\right) \simeq \operatorname{Hom}_{\mathcal{J}_{c}}\left(E, I_{q}^{\cdot}\right)
$$

Moreover, for any $k \in \mathbb{Z}$, we have

$$
\operatorname{Hom}_{\mathcal{T}_{c}}\left(E, I_{q}^{k}\right) \simeq \lim _{p \in P} \operatorname{Hom}_{\mathcal{T}_{c}}\left(E_{p}, I_{q}^{k}\right)
$$

Therefore,

$$
\begin{aligned}
\operatorname{RHom}_{\mathcal{T}_{c}}\left(E, F_{q}\right) \simeq \operatorname{Hom}_{\mathcal{T}_{c}}\left(E, I_{q}^{*}\right) & \simeq \lim _{p \in P} \operatorname{Hom}_{\mathcal{T}_{c}}\left(E_{p}, I_{q}^{\cdot}\right) \\
& \simeq \lim _{p \in P} \operatorname{RHom}_{\mathcal{T}_{c}}\left(E_{p}, F_{q}\right) .
\end{aligned}
$$

The conclusion follows.

Proposition 2.2.17. Let $\mathrm{J}$ be a small filtering category and let $E$ be an object of $\mathcal{T} c^{\mathcal{T}}$. Then, $E$ is $\underset{\hat{i} \in \mathrm{J}}{\mathrm{lim}-a c y c l i c}$ if and only if for any set $J$

$$
\operatorname{Hom}\left(E, l^{\infty}(J)\right): \mathcal{J}^{\text {op }} \rightarrow \mathcal{A} b
$$

is $\underset{i \in \mathrm{J}}{\lim \text {-acyclic. }}$ 
Proof. By [9, Proposition 3.6.3], for any set J, we have

$$
\mathrm{RHom}\left(\mathrm{L} \lim _{i \in \mathcal{J}} E(i), l^{\infty}(J)\right) \simeq \mathrm{R} \varliminf_{i \in J}\left(R H o m\left(E, l^{\infty}(J)\right)\right)(i) .
$$

Since $l^{\infty}(J)$ is an injective object of $\mathcal{T} c$, we obtain

$$
\operatorname{Hom}\left(\mathrm{L} \lim _{i \in \mathfrak{J}} E(i), l^{\infty}(J)\right) \simeq \mathrm{R} \lim _{i \in \mathfrak{J}}\left(\operatorname{Hom}\left(E, l^{\infty}(J)\right)\right)(i) .
$$

First, assume that $E$ is $\lim _{i \in \mathfrak{\jmath}}$-acyclic. For any set $J$, we get

$$
\begin{aligned}
\mathrm{R} \lim _{i \in \mathfrak{J}}\left(\operatorname{Hom}\left(E, l^{\infty}(J)\right)\right)(i) & \simeq \operatorname{Hom}\left(\lim _{i \in \mathfrak{J}} E(i), l^{\infty}(J)\right) \\
& \simeq \lim _{i \in \mathfrak{J}} \operatorname{Hom}\left(E, l^{\infty}(J)\right)(i) .
\end{aligned}
$$

Hence, $\operatorname{Hom}\left(E, l^{\infty}(J)\right)$ is $\underset{i \in \mathrm{J}}{\lim }$-acyclic.

Conversely, assume that for any set $J$, $\operatorname{Hom}\left(E, l^{\infty}(J)\right)$ is $\varliminf_{i \in J}$-acyclic. Then, if $k \neq 0$,

$$
H^{k}\left(\mathrm{R} \lim _{i \in J} \operatorname{Hom}\left(E, l^{\infty}(J)\right)(i)\right)=0 .
$$

Moreover,

$$
\begin{aligned}
\mathrm{R} \lim _{i \in \mathcal{J}} \operatorname{Hom}\left(E, l^{\infty}(J)\right)(i) & \simeq R^{\cdot}\left(\mathcal{J}, \operatorname{Hom}\left(E, l^{\infty}(J)\right)\right) \\
& \simeq \operatorname{Hom}\left(R .\left(\mathcal{J}^{\mathrm{op}}, E\right), l^{\infty}(J)\right)
\end{aligned}
$$

where the last isomorphism follows from [9, Lemma 3.6.2]. Therefore, if $k \neq 0$,

$$
H^{k}\left(\operatorname{Hom}\left(R .\left(\mathcal{J}^{\mathrm{op}}, E\right), l^{\infty}(J)\right)\right)=0,
$$

i.e. the complex

$$
\begin{aligned}
0 \rightarrow & \operatorname{Hom}\left(R_{0}\left(\mathcal{J}^{\mathrm{op}}, E\right), l^{\infty}(J)\right) \stackrel{\operatorname{Hom}\left(d_{1}, l^{\infty}(J)\right)}{\longrightarrow} \\
& \operatorname{Hom}\left(R_{1}\left(\mathcal{J}^{\mathrm{op}}, E\right), l^{\infty}(J)\right) \stackrel{\operatorname{Hom}\left(d_{2}, l^{\infty}(J)\right)}{\longrightarrow} \cdots
\end{aligned}
$$

is exact in degree $k \neq 0$.

If, for any $i \in \mathcal{J}$, we forget the topology of $E(i)$, then $E \in \mathrm{Ob}\left(\mathcal{A} b^{\mathrm{J}}\right)$. In this case, since the functor 


$$
\underset{i \in \mathfrak{J}}{\lim _{i \in \mathcal{J}}}: \mathcal{A} b^{\mathcal{J}} \rightarrow \mathcal{A} b
$$

is exact,

$$
\lim _{i \in \mathrm{J}} E(i) \simeq \mathrm{L} \underset{i \in \mathrm{J}}{\lim _{i \in \mathrm{J}}} E(i) \simeq R .\left(\mathcal{J}^{\mathrm{op}}, E\right) .
$$

So, the complex $R$. ( $\mathcal{J}^{\mathrm{op}}, E$ ) is algebraically exact in degree $k \geq 1$. Hence, Proposition 2.1 .13 shows that, for $k \geq 1$, the differential

$$
d_{k}: R_{k}\left(\mathcal{J}^{\mathrm{op}}, E\right) \rightarrow R_{k-1}\left(\mathcal{J}^{\mathrm{op}}, E\right)
$$

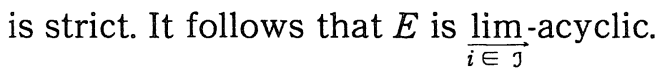

\section{§3. Separation Functors}

\section{§ 3.1. The Category $\widehat{\mathcal{T}}_{\mathfrak{C}}$}

Remark 3.1.1. Let $E$ be an object of $\mathcal{T} c$ with $P$ as system of semi-norms. Recall that $E$ is separated if

$$
\bigcap_{\substack{V \text { neighborhood } \\ \text { of zero }}} V=\{0\}
$$

or equivalently if the vanishing of $p(e)$ for any $p \in P$ implies $e=0$. Recall also that a generalized sequence $\left(x_{\alpha}\right)_{\alpha \in A}$ of $E$ is a family $\left(x_{\alpha}\right)_{\alpha \in A}$ of $E$ indexed by a filtering ordered set $A$. Such a sequence converges to a limit $x$ in $E$ if for any $\epsilon>0$ and any $p \in P$, there is $\alpha_{0} \in A$ such that

$$
p\left(x_{\alpha}-x\right) \leq \epsilon \quad \text { for } \alpha \geq \alpha_{0} \text {. }
$$

Clearly, a converging sequence $\left(x_{\alpha}\right)_{\alpha \in A}$ has a unique limit if $E$ is separated,.

Definition 3.1.2. We denote by $\widehat{\mathcal{T}}_{c}$ the full subcategory of $\mathcal{T}_{c}$ formed by separated spaces.

We have the following well-known facts:

Proposition 3.1.3. (i) Let $\left(E_{i}\right)_{i \in I}$ be a family of $\overline{\mathcal{T}} c$. Then, the locally convex spaces $\bigoplus_{i \in I} E_{i}$ and $\prod_{i \in I} E_{i}$ are separated. In particular, they 
form the direct sum and direct product of the family $\left(E_{i}\right)_{i \in I}$ in $\widehat{\mathcal{T}} c$.

(ii) Let $E$ be an object of $\mathcal{T} c$ and let $F$ be a vector subspace of $E$. Then, $E / F$ is separated if and only if $F$ is closed.

Proposition 3.1.4. Let $f: E \rightarrow F$ be a morphism of $\widetilde{\mathcal{T}} c$.

(i) The kernel of $f$ is the subspace $f^{-1}(0)$ of $E$ endowed with the induced topology.

(ii) The cokernel of $f$ is the quotient space $F / \overline{f(E)}$ endowed with the quotient topology.

(iii) The image of $f$ is the subspace $\overline{f(E)}$ of $F$ endowed with the induced topology.

(iv) The coimage of $f$ is the quotient space $E / f^{-1}(0)$ endowed with the quotient topology.

Corollary 3.1.5. Let $f: E \rightarrow F$ be a morphism of $\widetilde{\mathcal{T}}_{c}$. Then:

(i) $f$ is strict in $\widetilde{\mathcal{T}}_{c}$ if and only if $f$ is strict in $\mathcal{T}_{c}$ and has a closed range.

(ii) $f$ is a strict epimorphism of $\widehat{\mathcal{T}}_{C}$ if and only if $f$ is a strict epimorphism of $\mathcal{T} c$;

(iii) $f$ is a strict monomorphism of $\widehat{\mathcal{T}} c$ if and only if $f$ is a strict monomorphism of $\mathcal{T} c$ and has a closed range.

Lemma 3.1.6. A sequence

$$
0 \rightarrow E \stackrel{f}{\rightarrow} F \stackrel{g}{\rightarrow} G \rightarrow 0
$$

of $\widetilde{\mathcal{T}}_{c}$ is strictly exact if and only if it is strictly exact in $\mathcal{T}_{C}$.

Proof. (a) Assume that the sequence is strictly exact in $\widehat{\mathcal{T}} c$. We know that $(E, f)$ is a kernel of $g$ in $\mathcal{T} c$. Moreover, we have $G \simeq$ coker $f$ $\simeq F / \overline{f(E)}$. Since $f$ is strict in $\widehat{\mathcal{T}}_{C}, f(E)$ is closed and $G \simeq F / f(E)$. It follows that $(G, g)$ is a cokernel of $f$ in $\mathcal{T} C$.

(b) Conversely, assume that the sequence is strictly exact in $\mathcal{T} c$. On one hand, $(E, f)$ is a kernel of $g$ in $\widehat{\mathcal{T}} c$. On the other hand, in $\mathcal{T} c$, we have 


$$
f(E) \simeq \operatorname{im}(f) \simeq \operatorname{ker}(g) \simeq g^{-1}(0) \text {. }
$$

Since $G$ is separated, $g^{-1}(0)$ is closed. Hence, $f(E)$ is closed. Therefore, $(G, g)$ is a cokernel of $f$ in $\widehat{\mathcal{T}}_{c}$.

Lemma 3.1.7. Let $f: E \rightarrow F$ and $g: F \rightarrow G$ be two morphisms of

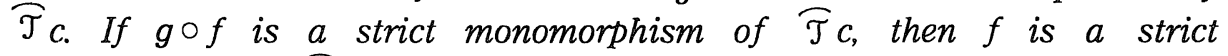
monomorphism of $\widehat{\mathcal{T}}$ c.

Proof. By Corollary 3.1.5, $g \circ f$ is a strict monomorphism of $\mathcal{T} c$. Then, we know that $f$ is a strict monomorphism of $\mathcal{T} c$. So, we only have to prove that $f$ has a closed range. Consider $y \in \overline{f(E)}$. There is a generalized sequence $\left(x_{\alpha}\right)_{\alpha \in A}$ of $E$ such that

$$
\left(f\left(x_{\alpha}\right)\right)_{\alpha \in A} \rightarrow y
$$

in $F$. It follows that

$$
\left((g \circ f)\left(x_{\alpha}\right)\right)_{\alpha \in A} \rightarrow g(y)
$$

in $G$ and that $g(y) \in \overline{(g \circ f)(E)}$. Since $g \circ f$ is strict in $\overline{\mathcal{T}}_{C, g}, g \circ$ has a closed range. Hence, there is $x \in E$ such that $g(y)=(g \circ f)(x)$. Therefore,

$$
\left((g \circ f)\left(x_{\alpha}\right)\right)_{\alpha \in A} \rightarrow(g \circ f)(x) \quad \text { in } G .
$$

Since $g \circ f$ is a strict monomorphism, $g \circ f: E \rightarrow g \circ f(E)$ is an isomorphism. Then, $\left(x_{\alpha}\right)_{\alpha \in A} \rightarrow x$ in $E$. Since $f$ is continuous, $\left(f\left(x_{\alpha}\right)\right)_{\alpha \in A}$ $\rightarrow f(x)$ in $F$ and since $F$ is separated, $y=f(x)$. Thus, we have obtained $\overline{f(E)} \subset f(E)$. The other inclusion being obvious, the conclusion follows.

Proposition 3.1.8. The category $\widehat{\mathcal{T}}_{c}$ is quasi-abelian.

Proof. We know that $\widehat{\mathcal{T}}_{c}$ is additive and that any morphism of $\widehat{\mathcal{T}}_{c}$ has a kernel and a cokernel.

Consider the cartesian square 


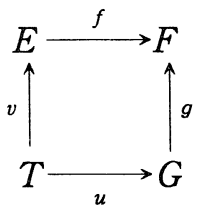

of $\overline{\mathcal{T}}_{c}$ where $f$ is a strict epimorphism. By Corollary $3.1 .5, f$ is a strict epimorphism of $\mathcal{T} c$. It follows that $u$ is a strict epimorphism of $\mathcal{T} c$ and hence of $\widehat{\mathcal{T}} c$.

Finally, consider the cocartesian square

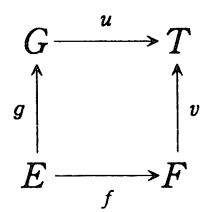

of $\mathcal{T}_{c}$ where $f$ is a strict monomorphism. Since

$$
\left(\begin{array}{ll}
0 & -1
\end{array}\right) \circ\left(\begin{array}{c}
g \\
-f
\end{array}\right)=f \text {, }
$$

Lemma 3.1.7 shows that $\left(\begin{array}{c}g \\ -f\end{array}\right): E \rightarrow G \oplus F$ is a strict monomorphism of $\overline{\mathcal{T}} c$. The square $\left(^{*}\right)$ being cocartesian, the sequence

$$
0 \longrightarrow E \stackrel{\left(\begin{array}{c}
g \\
-f
\end{array}\right)}{\longrightarrow} \oplus \oplus F \stackrel{\left(\begin{array}{ll}
u & v
\end{array}\right)}{\longrightarrow} T \longrightarrow 0
$$

is strictly exact in $\widehat{\mathcal{T}}_{c}$ and hence in $\mathcal{T}_{c}$ (see Lemma 3.1.6). It follows that the square $\left(^{*}\right)$ is cocartesian in $\mathcal{T} c$ and that $u$ is a strict monomorphism of $\tau c$. To conclude, let us prove that $u$ has a closed range. The morphisms $g$ and $v$ induce a strict quasi-isomorphism between the complexes

$$
0 \rightarrow E \stackrel{f}{\rightarrow} F \rightarrow 0 \quad \text { and } \quad 0 \rightarrow G \stackrel{u}{\rightarrow} T \rightarrow 0
$$

since the mapping cone of 


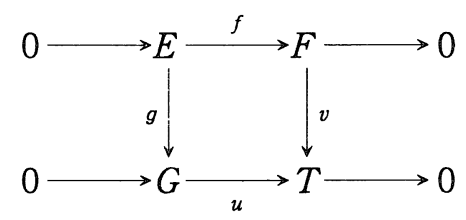

is the strictly exact complex $\left({ }^{* *}\right)$. Taking the cohomology and using the fact that $f$ and $u$ are strict monomorphisms, we get $\operatorname{coker}(f) \simeq \operatorname{coker}(u)$ in $\mathcal{T} c$. Since $f(E)$ is closed, $\operatorname{coker}(f)=F / f(E)$ is separated. It follows that coker $(u)=T / u(G)$ is separated and $u(G)$ is closed.

\section{§ 3.2. Equivalence between $\mathbb{D}\left(\mathcal{T}_{\mathbb{C}}\right)$ and $D\left(\widehat{\mathcal{T}}_{\mathbb{C}}\right)$}

Definition 3.2.1. We denote by

$$
\widehat{\mathrm{I}}: \widehat{\mathcal{T}}_{c} \rightarrow \mathcal{T}_{c}
$$

the inclusion functor and we define the functor

$$
\widehat{\text { Sep }:} \mathcal{T}_{c} \rightarrow \widehat{\mathcal{T}}_{c}
$$

by setting

$$
\widehat{\operatorname{Sep}}(E)=E / \overline{\{0\}}^{E}
$$

and endowing it with the quotient topology.

Proposition 3.2.2. For any object $E$ of $\mathcal{T}_{c}$ and any object $F$ of $\widehat{\mathcal{T}}_{c}$, we have the isomorphism

$$
\operatorname{Hom}_{\widehat{\mathcal{T}}_{c}}(\widehat{\operatorname{Sep}}(E), F) \simeq \operatorname{Hom}_{\mathcal{T}_{c}}(E, \widehat{\mathrm{I}}(F)) .
$$

Proof. This well-known isomorphism follows from the fact that if $f: E \rightarrow F$ is continuous and $F$ separated, then $f^{-1}(0)$ is a closed subset of $E$ containing 0 and hence $\overline{\{0\}}^{E}$.

Remark 3.2.3. Hereafter, $c^{0}$ denotes as usual the Banach space of complex sequences which converge to zero, endowed with the norm

$$
\left\|\left(x_{n}\right)_{n \in \mathbb{N}}\right\|_{c^{0}}=\sup _{n \in \mathbb{N}}\left|x_{n}\right| .
$$


Lemma 3.2.4. Denote by $\mathbb{C}^{-}$the space $\mathbb{C}$ endowed with the weakest topology. Then, there is a strictly exact sequence in $\mathcal{T} c$ of the form

$$
0 \rightarrow S_{1} \rightarrow S_{0} \rightarrow \mathbb{C}^{-} \rightarrow 0
$$

where $S_{0}$ and $S_{1}$ are separated.

Proof. Consider the subspace $S_{0}$ of $c^{0}$ formed by sequences $\left(x_{n}\right)_{n \in \mathbb{N}}$ such that $n x_{n}$ is constant for $n>>0$ and the subspace $S_{1}$ of $S_{0}$ formed by sequences $\left(x_{n}\right)_{n \in \mathbb{N}}$ null for $n>>0$. Since $c^{0}$ is a normed space, so are $S_{0}$ and $S_{1}$.

(a) Let us show that $S_{1}$ is dense in $c^{0}$. Consider $x=\left(x_{n}\right)_{n \in \mathbb{N}} \in c^{0}$ and $\epsilon>0$. There is $n_{0} \in \mathbb{N}$ such that $\left|x_{n}\right|<\epsilon$ for $n>n_{0}$. Define $y=$ $\left(y_{n}\right)_{n \in \mathbb{N}} \in S_{1}$ by setting

$$
y_{n}= \begin{cases}x_{n} & \text { if } n \leq n_{0} \\ 0 & \text { if } n>n_{0}\end{cases}
$$

Since $\|y-x\|_{c^{0}}=\sup _{n>n_{0}}\left|x_{n}\right| \leq \epsilon, S_{1}$ is dense in $c^{0}$.

(b) It follows from (a) that $S_{1}$ is dense in $S_{0}$. Hence, the quotient topology on $S_{0} / S_{1}$ is the weakest one. Let us show that $S_{0} / S_{1} \simeq \mathbb{C}$ as $\mathbb{C}$-vector spaces. Remark that the sequence $(1 / n)_{n \in \mathbb{N}}$ belongs to $S_{0} \backslash S_{1}$. Consider a sequence $x=\left(x_{n}\right)_{n \in \mathbb{N}}$ of $S_{0}$. There is $n_{0} \in \mathbb{N}$ and $c \in \mathbb{C}$ such that $n x_{n}=c$ for $n \geq n_{0}$. It follows that $x_{n}=c / n$ for $n \geq n_{0}$. If $y$ is the sequence of $S_{1}$ defined by

$$
y_{n}= \begin{cases}x_{n}-c / n & \text { if } n<n_{0} \\ 0 & \text { if } n \geq n_{0}\end{cases}
$$

then, $x=c(1 / n)_{n \in \mathbb{N}}+y$. Therefore, the class of $(1 / n)_{n \in \mathbb{N}}$ in $S_{0} / S_{1}$ forms a basis of this vector space.

(c) By (b), $S_{0} / S_{1}$ is isomorphic to $\mathbb{C}^{-}$. The sequence

$$
0 \rightarrow S_{1} \rightarrow S_{0} \rightarrow S_{0} / S_{1} \rightarrow 0
$$

being clearly strictly exact, the conclusion follows.

Proposition 3.2.5. For any object $E$ of $\mathcal{T} c$, there is a strictly exact sequence in $\mathcal{T} c$ of the form

$$
0 \rightarrow S_{1} \rightarrow S_{0} \rightarrow E \rightarrow 0
$$

where $S_{0}$ and $S_{1}$ are separated. 
Proof. We know that $E \simeq E / N \oplus N$ where $N$ is the closure of zero in $E$. Since in this formula $N$ is endowed with the weakest topology, we have $N \simeq \oplus_{b \in B} \mathbb{C}^{-}$where $B$ denotes a basis of $N$ as a $\mathbb{C}$-vector space. By Lemma 3.2.4, there is a strictly exact sequence in $\mathcal{T}_{C}$

$$
0 \rightarrow S_{1}^{\prime} \rightarrow S_{0}^{\prime} \rightarrow \mathbb{C}^{-} \rightarrow 0
$$

where $S_{0}^{\prime}$ and $S_{1}^{\prime}$ are separated. Since the sequence

$$
0 \rightarrow 0 \rightarrow E / N \stackrel{\text { id }}{\rightarrow} E / N \rightarrow 0
$$

is strictly exact and since direct sums are exact in $\mathcal{T} c$, the conclusion follows easily.

Lemma 3.2.6. The category $\widehat{\mathcal{T}} c$ is $\widehat{\text { Sep-projective. }}$

Proof. This follows directly from Proposition 3.2.5 and Lemma 3.1.6.

Proposition 3.2.7. (1) The functor $\widehat{\mathrm{I}}: \overparen{\mathcal{T}} c \rightarrow \quad \mathcal{T} c$ is kernel preserving and exact.

(2) The functor $\widehat{\text { Sep }:} \mathcal{T} c \rightarrow \widehat{\mathcal{T}}_{c}$ is cokernel preserving but not exact and it gives rise to a left derived functor

$$
\text { L } \widehat{\operatorname{Sep}}: D\left(\mathcal{T}_{c}\right) \rightarrow D\left(\widehat{\mathcal{T}}_{c}\right) \text {. }
$$

(3) The functor $\mathrm{L} \widehat{\mathrm{S}} \mathrm{ep}: D(\mathcal{T} c) \rightarrow D(\widehat{\mathcal{T}} c)$ is a quasi-inverse of $\widehat{\mathrm{I}}$ : $D\left(\widehat{\mathcal{T}}_{C}\right) \rightarrow D(\mathcal{T} c)$. In particular,

$$
D\left(\mathcal{T}_{c}\right) \approx D\left(\widehat{\mathcal{T}}_{c}\right)
$$

Proof. (1) Since the kernel of a morphism of $\widehat{\mathcal{T}}_{C}$ is the kernel of this morphism in $\mathcal{T}$, the functor $\mathrm{I}$ is kernel preserving. Moreover, by Lemma 3.1.6, $\widehat{\mathrm{I}}$ is exact.

(2) By the adjunction formula of Proposition 3.2.2, $\widehat{\text { Sep }}$ is cokernel preserving. It is not exact. As a matter of fact, let $E$ be a non closed subspace of the separated space $F$. The inclusion morphism $i: E \rightarrow F$ is a strict monomorphism of $\mathcal{T} c$. But $i=\widehat{\mathrm{Sep}}(i): E \rightarrow F$ is not a strict monomorphism of $\mathcal{T}_{c}$ since $i$ has not a closed range. By Lemma 3.2.6, Sep is left derivable.

(3) On one hand, for any object $S$ of $D\left(\widehat{\mathcal{T}}_{c}\right)$, we have 


$$
\text { L Sep } 0 \widehat{I}(S)=\mathrm{LSep}(\widehat{I}(S))=\widehat{\operatorname{Sep}}(\widehat{I}(S))=S
$$

where the second equality follows from the fact that the components of the complex $\widehat{I}(S)$ are separated. On the other hand, any object $E$ of $D(\mathcal{T} c)$ is quasi-isomorphic to a complex $S$ with separated components. Therefore, we have

$$
\mathrm{L} \widehat{\operatorname{Sep}}(E)=\widehat{\operatorname{Sep}}(S) \quad \text { and } \quad \widehat{\mathrm{I}} \circ \mathrm{L} \widehat{\operatorname{Sep}}(E) \simeq \widehat{\mathrm{I}} \circ \widehat{\operatorname{Sep}}(S) \simeq S \simeq E \text {. }
$$

\section{§ 3.3. The Functors Zcl and Sep}

Definition 3.3.1. We define the functors

$$
\mathrm{Zcl}: \mathcal{T} c \rightarrow \mathcal{T} c \text { and } \operatorname{Sep}: \mathcal{T} c \rightarrow \mathcal{T} c
$$

by setting

$$
\operatorname{Zcl}(E)=\overline{\{0\}}^{E} \quad \text { and } \quad \operatorname{Sep}(E)=E / \operatorname{Zcl}(E)
$$

where $\overline{\{0\}}^{E}$ is endowed with the weakest topology. Of course, we have

$$
\text { Sep }=\widehat{\mathrm{I}} \circ \widehat{\mathrm{Sep}} .
$$

Proposition 3.3.2. The functor Sep : $\mathcal{T} c \rightarrow \mathcal{T} c$ has a left derived functor

$$
\text { LSep : } D^{-}(\mathcal{T} c) \rightarrow D^{-}(\mathcal{T} c)
$$

which is equivalent to the identity functor.

Proof. Since $\widehat{\mathrm{I}}$ is exact, one has LSep $=\widehat{\mathrm{I}} \circ \mathrm{L} \widehat{\mathrm{Sep}}$ and the conclusion follows from Proposition 3.2.7.

Proposition 3.3.3. $\quad$ For any object $E$ of $\mathcal{T} c$, we have a distinguished triangle

$$
\mathrm{RZcl}(E) \rightarrow E \rightarrow \operatorname{RSep}(E) \stackrel{+1}{\rightarrow} .
$$

Proof. This follows directly from the fact that $\mathcal{T} c$ has enough 
injective objects and that for any object $E$ of $\mathcal{T} c$, the sequence

$$
0 \rightarrow \operatorname{Zcl}(E) \rightarrow E \rightarrow \operatorname{Sep}(E) \rightarrow 0
$$

is strictly exact.

\section{Proposition 3.3.4. Denote}

$$
(\cdot)^{-}: \nu \rightarrow \mathcal{T} c
$$

the functor which associates to any vector space $V$ the object of $\mathcal{T} c$ obtained by endowing $V$ with the weakest topology. Then, for any object $E$ of $\mathcal{T}_{c}$, we have

$$
\operatorname{Zcl}(E) \simeq\left(\operatorname{Hom}\left(\mathbb{C}^{-}, E\right)\right)^{-}
$$

In particular, $\mathrm{Zcl}: \mathcal{T}_{c} \rightarrow \mathcal{T}_{c}$ is a kernel preserving functor and, for any object $E$ of $\mathcal{T} c$, we have the isomorphism

$$
\operatorname{RZcl}(E) \simeq\left(\operatorname{RHom}\left(\mathbb{C}^{-}, E\right)\right)^{-}
$$

Proof. Let $E$ be an object of $\mathcal{T} c$. The first part follows from the fact if $f: \mathbb{C}^{-} \rightarrow E$ is a morphism of $\mathcal{T} c$, then $f^{-1}\left(\overline{\{0\}}{ }^{E}\right) \supset \overline{\{0\}} \mathbb{C}^{-}=\mathbb{C}^{-}$ and

$$
\operatorname{Hom}_{\mathcal{T}_{c}}\left(\mathbb{C}^{-}, E\right) \simeq \operatorname{Hom}_{v}(\mathbb{C}, \mathrm{Zcl}(E)) \simeq \operatorname{Zcl}(E)
$$

As for the second part, it follows from the fact that the functor Hom $\tau_{c}$ $\left(\mathbb{C}^{-},{ }^{\circ}\right): \mathcal{T} c \rightarrow \mathcal{V}$ is kernel preserving and that the functor $(\cdot)^{-}: \nu \rightarrow$ $\mathcal{T}_{c}$ is exact.

Proposition 3.3.5. For any family $\left(E_{i}\right)_{i \in I}$ of $\mathcal{T} c$, we have

$$
\operatorname{Zcl}\left(\prod_{i \in I} E_{i}\right) \simeq \prod_{i \in I} \operatorname{Zcl}\left(E_{i}\right) \quad \text { and } \quad \operatorname{Sep}\left(\prod_{i \in I} E_{i}\right) \simeq \prod_{i \in I} \operatorname{Sep}\left(E_{i}\right) .
$$

Proof. By definition of the functor $\mathrm{Zcl}$, we clearly have

$$
\operatorname{Zcl}\left(\prod_{i \in I} E_{i}\right) \simeq \prod_{i \in I} \operatorname{Zcl}\left(E_{i}\right)
$$

of $\mathcal{T} c$. Then, using the strictly exact sequences 


$$
0 \rightarrow \operatorname{Zcl}\left(E_{i}\right) \rightarrow E_{i} \rightarrow \operatorname{Sep}\left(E_{i}\right) \rightarrow 0
$$

we deduce easily from the first part that

$$
\operatorname{Sep}\left(\prod_{i \in I} E_{i}\right) \simeq \prod_{i \in I} \operatorname{Sep}\left(E_{i}\right)
$$

\section{$\S 4$. Completion Functors}

\section{§4.1. The Category $\widehat{\tau} c$}

Definition 4.1.1 Let $E$ be an object of $\mathcal{T} c$ with $P$ as system of semi-norms. A generalized sequence $\left(x_{\alpha}\right)_{\alpha \in A}$ of $E$ is a Cauchy sequence if for any $\epsilon>0$ and any $p \in P$, there is $\alpha_{0} \in A$ such that

$$
p\left(x_{\alpha}-x_{\alpha^{\prime}}\right) \leq \epsilon \quad \text { for } \alpha, \alpha^{\prime} \geq \alpha_{0} .
$$

An object $E$ of $\mathcal{T} c$ is complete if it is separated and if any Cauchy sequence of $E$ converges in $E$.

We denote by $\widehat{\mathcal{T}} c$ the full subcategory of $\mathcal{T} c$ formed by complete spaces.

Remark 4.1.2. Recall that to any object $E$ of $\mathcal{T} c$ is associated a complete object $\hat{E}$ and a canonical morphism $i_{E}: E \rightarrow \widehat{E}$ characterized by the fact that any morphism $f: E \rightarrow F$ with $F$ complete may be uniquely factored through $i_{E}$. Moreover, $i_{E}$ is a strict morphism whose image is dense in $\widehat{E}$ and whose kernel is the closure of zero in $E$.

As is well-known $\left[3\right.$, TG II, $\left.\S 3, n^{\circ} 7\right]$, the completion of an object of $\mathcal{T} c$ may also be characterized as follows:

Proposition 4.1.3. Let $j: E \rightarrow F$ be a morphism of $\mathcal{T} c$. If $F$ is complete, $j^{-1}(0)=\overline{\{0\}}^{E}, j$ is strict and $j(E)$ is dense in $F$, then for any morphism $g: E \rightarrow G$ with $G$ complete, there is a unique morphism $f: F \rightarrow G$ making the diagram

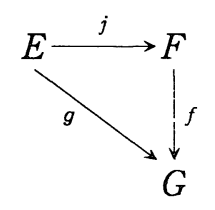


commutative,

Definition 4.1.4. We denote by

$$
\widehat{\mathrm{I}}: \widehat{\mathcal{T}}_{c} \rightarrow \mathcal{T} c
$$

the inclusion functor and we define the functor

$$
\widehat{\mathrm{C}} \mathrm{pl}: \mathcal{T} c \rightarrow \widehat{\mathcal{T}} c
$$

by setting $\widehat{C} \operatorname{pl}(E)=\widehat{E}$ where is $\widehat{E}$ is the complete separated space associated to $E$.

It follows from the definition of the completion of an object of $\mathcal{T}_{c}$ that:

Proposition 4.1.5. For any object $E$ of $\mathcal{T} c$ and any object $F$ of $\hat{\mathcal{T}}_{c}$, we have the isomorphism

$$
\operatorname{Hom}_{\widehat{\tau}_{c}}(\widehat{\mathrm{Cp}}(E), F) \simeq \operatorname{Hom}_{\mathcal{J}_{c}}(E, \widehat{\mathrm{I}}(F)) .
$$

For any object $F$ of $\widehat{\mathcal{T}} c$, the canonical morphism $\widehat{\mathrm{C}} \mathrm{pl} \circ \widehat{\mathrm{I}}(F) \rightarrow F$ is an isomorphism and for any object $E$ of $\mathcal{T} C$, the canonical morphism $i_{E}: E \rightarrow$ $\widehat{\mathrm{I}} \circ \widehat{\mathrm{C} p l}(E)$ is strict in $\mathcal{T} C$. Moreover, its image is dense and its kernel is the closure of zero in $E$. In particular, if $E$ is separated, $i_{E}$ is a strict monomorphism.

Proposition 4.1.6. (a) Any closed subspace of an object of $\widehat{\top}_{c}$ is complete.

(b) If $E$ is an object of $\widehat{T} c$ and if $F$ is a closed subspace of $E$, then the quotient space $E / F$ is not necessarily complete. (However, $E / F$ is a Fréchet space if $E$ is a Fréchet space).

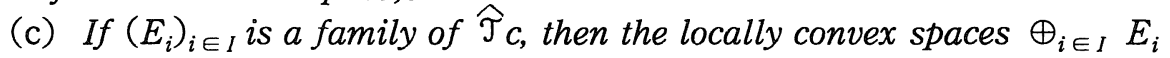
and $\Pi_{i \in I} E_{i}$ are complete. In particular, they form the direct sum and direct product of the family $\left(E_{i}\right)_{i \in I}$ in $\widehat{\mathcal{T}} c$.

Proof. (a) is clear.

(b) See for example [5, Problem 20D].

(c) See for example [6, Chap. I, §5,7.(2) (p. 37) and Chap. IV, § 18, 5.(3) (p. 212)]. 
Proposition 4.1.7. If $\left(E_{i}\right)_{i \in I}$ is a family of $\widehat{\tau} c$, we have

$$
\widehat{\mathrm{I}}\left(\bigoplus_{i \in I} E_{i}\right) \simeq \bigoplus_{i \in I} \widehat{\mathrm{I}}\left(E_{i}\right), \quad \widehat{\mathrm{I}}\left(\prod_{i \in I} E_{i}\right) \simeq \prod_{i \in I} \widehat{\mathrm{I}}\left(E_{i}\right)
$$

and if $\left(E_{i}\right)_{i \in I}$ is a family of $\mathcal{T} c$, we have

$$
\widehat{\mathrm{Cpl}}\left(\bigoplus_{i \in I} E_{i}\right) \simeq \bigoplus_{i \in I} \widehat{\mathrm{C}} \mathrm{pl}\left(E_{i}\right), \quad \widehat{\mathrm{Cpl}}\left(\prod_{i \in I} E_{i}\right) \simeq \prod_{i \in I} \widehat{\mathrm{C}} \mathrm{pl}\left(E_{i}\right) .
$$

Proof. This follows from Proposition 4.1.6 thanks to Proposition 4.1.3.

Proposition 4.1.8. Let $f: E \rightarrow F$ be a morphism of $\widehat{\tau} c$.

(i) The kernel of $f$ is the subspace $f^{-1}(0)$ of $E$ endowed with the induced topology.

(ii) The cokernel of $f$ is the space $\widehat{F / f(E)}$ where $F / f(E)$ is endowed with the quotient topology.

(iii) The image of $f$ is the subspace $\overline{f(E)}$ of $F$ endowed with the induced topology.

(iv) The coimage of $f$ is the space $E / \widehat{f^{-1}}(0)$ where $E / f^{-1}(0)$ is endowed with the quotient topology.

Proposition 4.1.9. Let $f: E \rightarrow F$ be a morphism of $\widehat{T}_{c}$. Then, $f$ is strict in $\hat{\mathcal{T}} c$ if and only if $f$ is strict in $\mathcal{T} c$.

Proof. First, assume that $f$ is strict in $\widehat{\mathcal{T}} c$. Hence, the canonical morphism

$$
\varphi: E / \widehat{f^{-1}}(0) \rightarrow \overline{f(E)}
$$

is an isomorphism. Consider the commutative diagram of $\mathcal{T}_{c}$

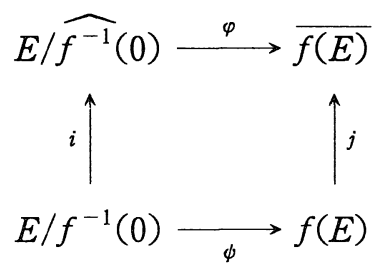

where $i$ and $\phi$ are the canonical morphisms and $j$ the inclusion morphism. Since $E$ is separated and $f^{-1}(0)$ is closed, $E / f^{-1}(0)$ is separated. Then, by Proposition 4.1.5, $i$ is a strict monomorphism of $\mathcal{T} c$. Therefore, $j \circ \phi=\varphi \circ i$ is a strict monomorphism of $\mathcal{T} c$. It follows that $\phi$ is a strict monomorphism. Since $\phi$ is clearly an epimorphism, $\phi$ is an 
isomorphism of $\mathcal{T} c$. Hence, $f$ is strict in $\mathcal{T} c$.

Conversely, assume that $f$ is strict in $\mathcal{T} c$, i.e. $\phi$ is an isomorphism. Since $f(E)$ and $\overline{f(E)}$ are endowed with the topology induced by that of $F, j$ is a strict monomorphism. It follows that $j \circ \phi$ is a strict monomorphism. Moreover, the image of $j \circ \phi$ is dense and since $\overline{f(E)}$ is a closed subspace of the complete space $F, \overline{f(E)}$ is also complete. Then, by Proposition 4.1.3, there is a unique morphism

$$
\varphi^{\prime}: \overline{f(E)} \rightarrow E / \widehat{f^{-1}}(0)
$$

making the diagram

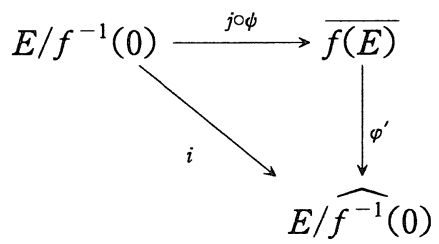

commutative. One checks easily that $\varphi$ and $\varphi^{\prime}$ are inverse one of each other. The conclusion follows.

Proposition 4.1.10. Let $f: E \rightarrow F$ be a morphism of $\widehat{\tau} c$. Then,

(i) $f$ is a monomorphism (resp. strict monomorphism) of $\widehat{\tau} c$ if and only if $f$ is a monomorphism (resp. strict monomorphism) of $\mathcal{J}_{c}$;

(ii) $f$ is an epimorphism (resp. strict epimorphism) of $\widehat{\tau}_{c}$ if and only if $f(E)$ is dense in $F$ (resp. $f(E)$ is dense in $F$ and $f$ is strict in $\mathcal{T} c$ ).

Proof. This follows directly from Proposition 4.1.8 and Proposition 4.1.9.

Remark 4.1.11. (i) If $f: E \rightarrow F$ is a strict monomorphism of $\widehat{\top} c$, then $f(E)$ is closed. As a matter of fact, if $f$ is a strict monomorphism, the canonical morphism $f: E \rightarrow \overline{f(E)}$ is an isomorphism. In particular, $f(E)$ $=\overline{f(E)}$.

(ii) If $f: E \rightarrow F$ is a strict epimorphism of $\widehat{\mathcal{T}}_{c}$, then $f$ is not necessarily an epimorphism of $\mathcal{T} c$. As a matter of fact, let $E$ be an object of $\widehat{T} c$ and $N$ a closed subspace of $E$ such that $E / N$ is not complete. Set $F$ $=\widehat{E / N}$. If $q: E \rightarrow E / N$ and $i: E / N \rightarrow \widehat{E / N}$ are the canonical morphisms, set $f=i \circ q: E \rightarrow F$. Since $f(E)$ is dense in $F$, by Proposition 4.1.10, $f$ is an epimorphism of $\hat{\mathcal{J}} c$. Hence, 


$$
\operatorname{im}(f)=F \quad \text { in } \widehat{\jmath} c .
$$

The subspace $N$ being closed, $E / N$ is separated and by Proposition 4.1.5, $i$ is injective. It follows that

$$
f^{-1}(0)=(i \circ q)^{-1}(0)=q^{-1}\left(i^{-1}(0)\right)=q^{-1}(0)=N
$$

and that

$$
\operatorname{coim}(f) \simeq \widehat{E / N} \simeq F \quad \text { in } \widehat{\mathcal{T}} c .
$$

By $\left({ }^{*}\right)$ and $\left({ }^{* *}\right), f$ is strict in $\widehat{\top} c$. But, since $E / N$ is not complete, $f(E) \neq F$. So, $f$ is not an epimorphism of $\mathcal{T} c$.

Proposition 4.1.12. If $u: E \rightarrow F$ is a strict monomorphism of $\mathcal{T} c$, then, $\hat{u}: \widehat{E} \rightarrow \widehat{F}$ is a strict monomorphism of $\mathcal{T} c$ and, hence, of $\widehat{\mathcal{T}} c$.

Proof. See e.g. [3, TG II, p. 26, cor. 1]

Proposition 4.1.13. The functor $\widehat{\mathrm{I}}: \widehat{\mathcal{T}} c \rightarrow \tau c$ is kernel preserving, but not exact. The functor $\widehat{\mathrm{C}} \mathrm{pl}: \mathcal{T} c \rightarrow \widehat{\mathcal{T}} c$ is cokernel preserving and exact, but not kernel preserving.

Proof. The fact that $\widehat{\mathrm{I}}$ (resp. $\widehat{\mathrm{C}} \mathrm{pl}$ ) is kernel (resp. cokernel) preserving follows from the adjunction formula between $\widehat{\mathrm{I}}$ and $\widehat{\mathrm{C}} \mathrm{pl}$.

Let us show that $\widehat{\mathrm{I}}$ is not exact. If $E$ is an object of $\widehat{\tau} c$ and $F$ a closed subspace of $E$ such that $E / F$ is not complete, the sequence

$$
0 \rightarrow F \rightarrow E \rightarrow \widehat{E / F} \rightarrow 0
$$

is strictly exact in $\widehat{\mathcal{T}} c$ but not in $\mathcal{T} c$ (see Remark 4.1.11).

Now, let us prove that $\widehat{\mathrm{C}} \mathrm{pl}$ is exact. Consider a strictly exact sequence

$$
0 \rightarrow E \stackrel{u}{\rightarrow} F \stackrel{v}{\rightarrow} G \rightarrow 0
$$

of $\mathcal{T} c$. Since $u: E \rightarrow F$ is a strict monomorphism of $\mathcal{T} c$, by Proposition 4.1.12, $\widehat{u}: \widehat{E} \rightarrow \widehat{F}$ is a strict monomorphism. It follows that $\widehat{u}$ is the kernel of its cokernel. Moreover, since $\widehat{C} p l$ is cokernel preserving, $\widehat{v}$ is the cokernel of $\hat{u}$. Therefore, the sequence 


$$
0 \rightarrow \widehat{\hat{u}} \rightarrow \widehat{\hat{F}} \stackrel{\hat{v}}{\rightarrow} \widehat{G} \rightarrow 0
$$

is strictly exact in $\widehat{\mathcal{T}} c$.

Finally, let us show that $\widehat{\mathrm{C}} \mathrm{pl}$ is not kernel preserving. Consider a vector space $V \neq 0$ and denote by $V^{+}$(resp. $V^{-}$) the object $V$ of $\mathcal{T} c$ endowed with the strongest (resp. weakest) locally convex topology. Then, the identity map $\varphi: V^{+} \rightarrow V^{-}$is continuous and we have $\operatorname{ker}(\varphi)=0$. Since $\hat{V}^{-}=0$, we have $\operatorname{ker}(\hat{\varphi})=\widehat{V}^{+}$. Moreover, since $V^{+}$is separated, by Proposition 4.1.5, $i_{V^{+}}: V^{+} \rightarrow \hat{V}^{+}$is injective and $i_{V^{+}}\left(V^{+}\right) \neq 0$. It follows that

$$
\operatorname{ker}(\hat{\varphi})=\hat{V}^{+} \supset i_{V^{+}}\left(V^{+}\right) \neq 0,
$$

and $\widehat{\mathrm{C}} \mathrm{pl}$ is not kernel preserving.

Proposition 4.1.14. The category $\widehat{\mathcal{T}} c$ is not quasi-abelian.

Proof. Let $F$ be a closed subspace of an object $E$ of $\widehat{\mathcal{T}} c$ such that $E / F$ is not complete. Set $G=\widehat{E / F}$. If $q: E \rightarrow E / F$ and $i: E / F \rightarrow \widehat{E / F}$ are the canonical morphisms, set $f=i \circ q: E \rightarrow G$. By Remark 4.1.11, $f$ is a strict epimorphism of $\widehat{\mathcal{T}}_{c}$ and $f(E) \neq G$. Fix $x \in G \backslash f(E)$. Consider the continuous linear map $m: \mathbb{C} \rightarrow G$ defined by $m(c)=c x$ for $c \in \mathbb{C}$. Let us show that the commutative square

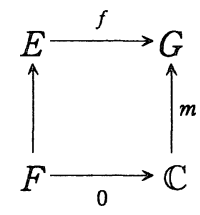

is cartesian in $\widehat{\mathcal{T}} c$. Consider $e \in E$ and $c \in \mathbb{C}$ such that

$$
\left(\begin{array}{ll}
f & -m
\end{array}\right)\left(\begin{array}{l}
e \\
c
\end{array}\right)=f(e)-m(c)=0 .
$$

Hence, $f(e)=c x$. If $c \neq 0$, then $x=f(e / c)$. Since $x \in G \backslash f(E)$, we get a contradiction. It follows that $c=0$ and $f(e)=0$. Since by Remark 4.1.11, $f^{-1}(0)=F$ we get $e \in F$. Therefore, we have successively

$$
(f-m)^{-1}(0)=\{(e, c): e \in F, c=0\} \simeq F
$$

and the square $\left(^{*}\right)$ is cartesian. Since $\{0\}$ is not dense in $\mathbb{C}$, by Proposition 4.1.10, $0: F \rightarrow \mathbb{C}$ is not a strict epimorphism of $\widehat{\mathcal{T}} c$. Hence, 
the conclusion.

\section{$\S$ 4.2. The Functor $\mathrm{Cpl}$ and Cohomological Completeness}

Definition 4.2.1. We define the functor

$$
\mathrm{Cpl}: \mathcal{T}_{C} \rightarrow \mathcal{T} c
$$

by setting

$$
\mathrm{Cpl}=\widehat{\mathrm{I}} \circ \widehat{\mathrm{C}} \mathrm{pl}
$$

Proposition 4.2.2. The functor $\mathrm{Cpl}$ is left exact and has a right derived functor

$$
\mathrm{RCpl}: D^{+}(\mathcal{T} c) \rightarrow D^{+}(\mathcal{T} c)
$$

Proof. By Proposition 4.1.13, the functor $\widehat{\mathrm{I}}$ is kernel preserving and the functor $\widehat{\mathrm{C} p l}$ is exact. Then, the functor $\mathrm{Cpl}=\widehat{\mathrm{I}} \circ \widehat{\mathrm{C}} \mathrm{pl}$ is left exact. Since $\mathcal{T} c$ has enough injective objects, $\mathrm{Cpl}$ is right derivable.

Definition 4.2.3. An object $E^{\cdot}$ of $D^{+}(\mathcal{T} c)$ is cohomologically complete if

$$
\operatorname{RCpl}\left(E^{\cdot}\right) \simeq E^{\cdot}
$$

in $D^{+}(\mathcal{T} c)$. We denote by $D_{c c}^{+}(\mathcal{T} c)$ the full subcategory of $D^{+}(\mathcal{T} c)$ formed by cohomologically complete complexes.

Proposition 4.2.4. The category $D_{c c}^{+}(\mathcal{T} c)$ is a triangulated subcategory of the derived category $D^{+}(\mathcal{T} c)$.

Proof. Consider a distinguished triangle $E^{\prime} \rightarrow E \rightarrow E^{\prime \prime} \stackrel{+1}{\rightarrow}$ of $D^{+}$ $\left(\mathcal{T} C\right.$ ) such that $E^{\prime}$ and $E$ are cohomologically complete. Since the functor $\mathrm{RCpl}$ is triangulated, the triangle

$$
\operatorname{RCpl}\left(E^{\prime}\right) \rightarrow \operatorname{RCpl}(E) \rightarrow \operatorname{RCpl}\left(E^{\prime \prime}\right) \stackrel{+1}{\rightarrow}
$$

is distinguished in $D^{+}(\mathcal{T} c)$. Moreover, we have the morphism of distinguished triangles 


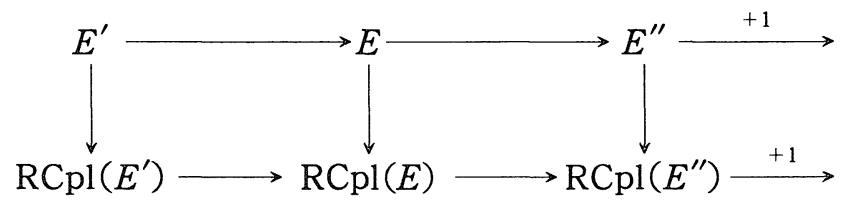

The complexes $E$ and $E^{\prime}$ being cohomologically complete, we have

$$
\operatorname{RCpl}(E) \simeq E \quad \text { and } \quad \operatorname{RCpl}\left(E^{\prime}\right) \simeq E^{\prime} .
$$

If follows that $\operatorname{RCpl}\left(E^{\prime \prime}\right) \simeq E^{\prime \prime}$ and that $E^{\prime \prime}$ is cohomologically complete. The conclusion follows easily.

Proposition 4.2.5. For any object $E^{*}$ of $D^{+}\left(T_{C}\right)$, the object $\mathrm{RCpl}\left(E^{*}\right)$ is cohomologically complete. In particular, $\mathrm{RCpl}$ induces a functor

$$
\mathrm{RCpl}: D^{+}(\mathcal{T} c) \rightarrow D_{c c}^{+}(\mathcal{T} c)
$$

which is a left quasi-inverse of the inclusion functor

$$
D_{c c}^{+}(\mathcal{T} c) \rightarrow D^{+}(\mathcal{T} c)
$$

Proof. We know that $E^{*}$ is quasi-isomorphic to a complex $I^{*}$ such that each $I^{k}$ is of the type

$$
V_{k} \times \prod_{j_{k} \in J_{k}} F_{j_{k}}
$$

where $V_{k}$ is a vector space endowed with the weakest topology and $F_{j_{k}}$ is an injective Banach space. Since $\operatorname{Cpl}\left(I^{k}\right) \simeq \Pi_{j_{k} \in J_{k}} F_{j_{k^{\prime}}} \operatorname{Cpl}\left(I^{k}\right)$ is an injective object of $\mathcal{T} c$ which is complete. Therefore,

$$
\operatorname{RCpl}(\operatorname{RCpl}(E)) \simeq \operatorname{RCpl}\left(\operatorname{Cpl}\left(I^{*}\right)\right) \simeq \operatorname{Cpl}\left(\operatorname{Cpl}\left(I^{*}\right)\right) \simeq \operatorname{Cpl}\left(I^{*}\right) \simeq \operatorname{RCpl}(E) .
$$

Hence, the conclusion.

Proposition 4.2.6. The product of cohomologically complete spaces is a cohomologically complete space.

Proof. Let $\left(E_{j}\right)_{j \in J}$ be a family of cohomologically complete spaces. For any $j \in J$, let $I_{j}^{\prime}$ be an injective resolution of $E_{j}$ in $\mathcal{T}_{C}$. Since products are exact in $\mathcal{T} c$ and since the product of injective objects in an injective 
object, $\Pi_{j \in J} I_{j}^{\prime}$ is an injective resolution of $\prod_{j \in J} E_{j}$. It follows that

$$
\operatorname{RCpl}\left(\prod_{j \in J} E_{j}\right) \simeq \operatorname{Cpl}\left(\prod_{j \in J} I_{j}^{*}\right) \simeq \prod_{j \in J} \operatorname{Cpl}\left(I_{j}^{*}\right) \simeq \prod_{j \in J} \operatorname{RCpl}\left(E_{j}\right) \simeq \prod_{j \in J} E_{j}
$$

where the second isomorphism follows from Proposition 4.1.7.

Proposition 4.2.7. If $E$ is a cohomologically complete object of $\mathcal{T} c$, then $E$ is complete.

Proof. Since $E \simeq \mathrm{RCpl}(E)$ and since $\mathrm{Cpl}$ is left exact, we have

$$
E \simeq L H^{0}(E) \simeq L H^{0}(\operatorname{RCpl}(E)) \simeq \operatorname{Cpl}(E) .
$$

Remark 4.2.8. Complete objects of $\mathcal{T} c$ are not always cohomologically complete. For example consider a complete space $E$ and a closed subspace $F$ of $E$ such that the quotient space $E / F$ in not complete. Then, the sequence

$$
0 \rightarrow F \rightarrow E \rightarrow E / F \rightarrow 0
$$

is strictly exact and gives rise to the distinguished triangle

$$
F \rightarrow E \rightarrow E / F \stackrel{+1}{\rightarrow}
$$

Assume that $E$ and $F$ are cohomologically complete. By Proposition 4.2.4, $E / F$ is cohomologically complete and then complete. Hence, a contradiction.

Proposition 4.2.9. For any object $E$ of $\mathcal{T} c$, we have a canonical isomorphism

$$
\mathrm{RSep}(E) \simeq \operatorname{RCpl}(E) .
$$

In particular,

$$
L H^{0}(\mathrm{RSep}(\mathrm{E})) \simeq \operatorname{Cpl}(E) .
$$

Proof. Let $E$ be an object of $\mathcal{T} c$ and let $I^{*}$ be an injective resolution of $E$. For any $n$, we may assume that 


$$
I^{n}=V^{n} \times \prod_{j_{n} \in J_{n}} B^{j_{n}}
$$

where $V^{n}$ is an object of $\mathcal{T} c$ with the weakest topology and $B^{j_{n}}$ is an injective Banach space for any $j_{n} \in J_{n}$. Then, we have successively

$$
\operatorname{Cpl}\left(I^{n}\right) \simeq \operatorname{Cpl}\left(V^{n}\right) \times \prod_{j_{n} \in J_{n}} \operatorname{Cpl}\left(B^{j_{n}}\right) \simeq \prod_{j_{n} \in J_{n}} B^{j_{n}}
$$

and

$$
\operatorname{Sep}\left(I^{n}\right) \simeq \operatorname{Sep}\left(V^{n}\right) \times \prod_{j_{n} \in J_{n}} \operatorname{Sep}\left(B^{j_{n}}\right) \simeq \prod_{j_{n} \in J_{n}} B^{j_{n}} .
$$

If follows that

$$
\mathrm{RCpl}(E) \simeq \operatorname{Cpl}\left(I^{*}\right) \simeq \operatorname{Sep}\left(I^{*}\right) \simeq \mathrm{RSep}(E) .
$$

Proposition 4.2.10. Let $E$ be an object of $\mathcal{T} c$.

(i) $E$ is separated $\Longleftrightarrow \operatorname{Zcl}(E) \simeq 0$.

(ii) $E$ is complete $\Longleftrightarrow \mathrm{Zcl}(E) \simeq 0$ and $L H^{1}(\operatorname{RZcl}(E)) \simeq 0$.

(iii) $E$ is cohomologically complete $\Longleftrightarrow \mathrm{RZcl}(E) \simeq 0$.

Proof. (i) is clear.

(ii) By Proposition 3.3.3 and Proposition 4.2.9, we have the distinguished triangle

$$
\operatorname{RZcl}(E) \rightarrow E \rightarrow \operatorname{RCpl}(E) \stackrel{+1}{\rightarrow}
$$

Since the functors $\mathrm{Zcl}$ and $\mathrm{Cpl}$ are left exact, we have the long exact sequence

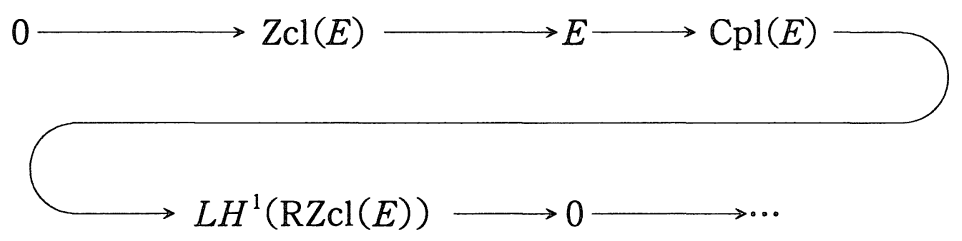

So, $\operatorname{Cpl}(E) \simeq E$ if and only if $\operatorname{Zcl}(E) \simeq 0$ and $L H^{1}(\operatorname{RZcl}(E)) \simeq 0$. The conclusion follows.

(iii) By definition, $E$ is cohomologically complete if and only if $E \simeq$ 
$\mathrm{RCpl}(E)$. Since the triangle $\left({ }^{*}\right)$ is distinguished, $E$ is cohomologically complete if and only if $\operatorname{RZcl}(E) \simeq 0$.

Corollary 4.2.11. Let $E$ be an object of $\mathcal{T} c$. Then,

(i) $E$ is separated $\Longleftrightarrow \operatorname{Hom}\left(\mathbb{C}^{-}, E\right) \simeq 0$.

(ii) $E$ is complete $\Longleftrightarrow \operatorname{Hom}\left(\mathbb{C}^{-}, E\right) \simeq 0$ and $\operatorname{Ext}^{1}\left(\mathbb{C}^{-}, E\right) \simeq 0$.

(iii) $E$ is cohomologically complete $\Longleftrightarrow \mathrm{RHom}\left(\mathbb{C}^{-}, E\right) \simeq 0$.

Proof. (i) follows from Proposition 4.2.10 and Proposition 3.3.4.

(ii) By Proposition 4.2.10, $E$ is complete if and only if

$$
\operatorname{Zcl}(E) \simeq 0 \quad \text { and } \quad L H^{1}(\operatorname{RZcl}(E)) \simeq 0 .
$$

We have $\operatorname{Zcl}(E) \simeq\left(\operatorname{Hom}\left(\mathbb{C}^{-}, E\right)\right)^{-}$and

$$
L H^{1}(\mathrm{RZcl}(E)) \simeq\left[H^{1}\left(\mathrm{RHom}\left(\mathbb{C}^{-}, E\right)\right)\right]^{-} \simeq \operatorname{Ext}^{1}\left(\mathbb{C}^{-}, E\right)^{-}
$$

where the first isomorphism follows from the fact that any morphism between objects of $\tau c$ with the weakest topology is strict. Hence, the conclusion.

(iii) follows from Proposition 4.2.10.

\section{§4.3. Equivalence between $D_{c c}^{+}(\mathcal{T} c)$ and $D^{+}(\mathcal{P} r o(\mathcal{B} a n))$}

Definition 4.3.1. The category of Banach spaces is the full subcategory of $\tau c$ whose objects are the Banach spaces. We denote it by Ban.

One can show that the category $\mathcal{B}$ an is a quasi-abelian category with enough injective objects. Moreover, the space $l^{1}(I)$ of summable sequences of $\mathbb{C}$ indexed by $I$ is projective and $\mathcal{B} a n$ has enough projective objects. For more details, see [8].

Hereafter, we consider the category $\operatorname{Pro}(\mathrm{B} a n)$ of pro-objects of Ban. Recall that the objects of $\mathcal{P} r o(B a n)$ are functors

$$
E: \mathcal{J}^{\mathrm{op}} \rightarrow \mathcal{B} \text { an }
$$

where $\mathcal{J}$ is a small filtering category and that if 


$$
\mathrm{E}: \mathcal{J}^{\mathrm{op}} \rightarrow \mathcal{B} \text { an, } \quad F: \mathrm{J}^{\mathrm{op}} \rightarrow \mathrm{B} \text { an }
$$

are two such functors, then

$$
\operatorname{Hom}_{\mathcal{P r o}(\mathcal{B} a n)}(E, F)=\lim _{j \in \delta} \lim _{i \in \mathcal{J}} \operatorname{Hom}_{\mathcal{B} a n}(E(i), F(j)) .
$$

For further details on pro-objects, we refer the reader to classical sources (such as $[1,2]$ ) and to [9] for the main results in the context of quasi-abelian categories. Following the standard usage and to avoid confusions, we will denote

$$
\text { “ } \lim _{i \in \mathrm{J}} ” E(i)
$$

the functor $E: \mathcal{J}^{\text {op }} \rightarrow \mathcal{B}$ an considered as an object of $\operatorname{Pro}(\mathcal{B} a n)$. Similarly, we denote " $X$ " the pro-object associated to the Banach space $X$. In other words, we set

$$
" X "=“ \lim _{i \in J} " C(i)
$$

where $\mathcal{J}$ is a one point category anc $C: \mathcal{J}^{\text {op }} \rightarrow \mathcal{B}$ an is the constant functor with value $X$.

Applying the results of [9], we get:

Proposition 4.3.2. The category $\operatorname{Pro}(\mathcal{B}$ an $)$ is a complete quasiabelian category with exact filtering projective limits.

Proposition 4.3.3. A sequence

$$
E \stackrel{u}{\rightarrow} F \stackrel{v}{\rightarrow} G
$$

of $\operatorname{Pro}(\mathcal{B} a n)$ is costrictly exact if and only if the sequence

$$
\begin{aligned}
\operatorname{Hom}_{\mathcal{P} r o(\mathcal{B} a n)}\left(G, " l^{\infty}(I) "\right) & \rightarrow \operatorname{Hom}_{\mathcal{P} r o(\mathcal{B} a n)}\left(F, " l^{\infty}(I) "\right) \\
& \rightarrow \operatorname{Hom}_{\mathcal{P r o}(\mathcal{B} a n)}\left(E, “ l^{\infty}(I) "\right)
\end{aligned}
$$

is exact for any set I

Proof. $\quad$ Work as in Proposition 2.1.13. 
Proposition 4.3.4. For any object $E$ of $\mathcal{T} c$, the functor

$$
\mathrm{S}^{\prime}(E): \mathcal{B} a n \rightarrow \mathcal{A} b
$$

defined by setting

$$
\mathrm{S}^{\prime}(E)(X)=\operatorname{Hom}_{\tau_{c}}(E, X)
$$

is pro-represented by “ $\lim _{p \in P}$ " $\widehat{E}_{p}$, where $P$ is the system of semi-norms of $E$.

Proof. Let $E$ be an object of $\mathcal{T}_{c}$ with $P$ as system of semi-norms. For any Banach space $X$, we get successively

$$
\begin{aligned}
\mathrm{S}^{\prime}(E)(X) & \simeq \operatorname{Hom}_{\tau_{c}}(E, X) \simeq \lim _{p \in P} \operatorname{Hom}_{\tau_{c}}\left(E_{p}, X\right) \\
& \simeq \varliminf_{p \in P} \operatorname{Hom}_{\mathcal{B} a n}\left(\widehat{E}_{p}, X\right) \simeq \operatorname{Hom}_{\mathcal{P r o}(\operatorname{Ban})}\left({ }^{\prime \lim _{p \in P}} " \widehat{E}_{p}, " X^{n}\right)
\end{aligned}
$$

where the second isomorphism follows from Lemma 2.2.15.

Definition 4.3.5. Let

$$
\mathrm{S}: \mathcal{I} c \rightarrow \operatorname{P} r o(\operatorname{Ban})
$$

be the functor characterized by the isomorphism

$$
\operatorname{Hom}_{\operatorname{Pro}(\mathcal{B} a n)}(\mathrm{S}(E), " X ") \simeq \operatorname{Hom}_{\tau_{c}}(E, X)
$$

where $X$ is in Ban and $E$ in $\mathcal{T} c$.

Remark 4.3.6. For any object $E$ of $\mathcal{T} C$ with $P$ as system of seminorms, we have

$$
\mathrm{S}(E) \simeq “ \lim _{p \in P} " \widehat{E}_{p} .
$$

In particular, if $E$ is a semi-normed space, then

$$
\mathrm{S}(E) \simeq \text { " }
$$

Proposition 4.3.7. For any object $E$ of $\mathcal{T} c$, we have

$$
\mathrm{S}(E) \simeq \mathrm{S}(\widehat{E}) .
$$


Proof. Let $E$ be an object of $\mathcal{T} c$ with $P$ as system of semi-norms. For any Banach space $X$, we have successively

$$
\begin{aligned}
& \operatorname{Hom}_{\mathcal{P r o}_{(\mathcal{B} a n)}}(\mathrm{S}(E), “ X ") \simeq \operatorname{Hom}_{\mathcal{T}_{c}}(E, X) \simeq \operatorname{Hom}_{\mathcal{T} c}(\widehat{E}, X) \\
& \simeq \operatorname{Hom}_{\mathcal{P}_{r o}(\mathcal{B} a n)}(\mathrm{S}(\widehat{E}), " X ") \text {. }
\end{aligned}
$$

Proposition 4.3.8. If $\left(E_{i}\right)_{i \in I}$ is a small family of $\mathcal{T} c$, then

$$
\mathrm{S}\left(\prod_{i \in I} E_{i}\right) \simeq \prod_{i \in I} \mathrm{~S}\left(E_{i}\right)
$$

Proof. For any Banach space $X$, we have

$$
\begin{aligned}
\operatorname{Hom}_{\mathcal{P} r o(\mathcal{B} a n)}\left(\mathrm{S}\left(\prod_{i \in I} E_{i}\right), “ X "\right) & \simeq \operatorname{Hom}_{\tau_{c}}\left(\prod_{i \in I} E_{i}, X\right) \simeq \bigoplus_{i \in I} \operatorname{Hom}_{\mathcal{T}_{c}}\left(E_{i}, X\right) \\
& \simeq \bigoplus_{i \in I} \operatorname{Hom}_{\mathcal{P} r o(\mathcal{B} a n)}\left(\mathrm{S}\left(E_{i}\right), “ X "\right) \\
& \simeq \operatorname{Hom}_{\mathcal{P} r o(\mathcal{B} a n)}\left(\prod_{i \in I} \mathrm{~S}\left(E_{i}\right), “ X ”\right)
\end{aligned}
$$

where the second isomorphism follows from Proposition 2.1.6.

Proposition 4.3.9. If

$$
X \rightarrow Y \rightarrow Z
$$

is a costrictly exact sequence of $\mathcal{T} c$, then the sequence

$$
\mathrm{S}(X) \rightarrow \mathrm{S}(Y) \rightarrow \mathrm{S}(Z)
$$

is costrictly exact in $\operatorname{Pro}(\mathcal{B} a n)$. In particular, the functor $S$ is exact and cokernel preserving.

Proof. Let $X \rightarrow Y \rightarrow Z$ be a costrictly exact sequence of $\mathcal{T}$ c. By Proposition 2.1.13, the sequence

$$
\operatorname{Hom}_{\tau_{c}}\left(Z, l^{\infty}(I)\right) \rightarrow \operatorname{Hom}_{\tau_{c}}\left(Y, l^{\infty}(I)\right) \rightarrow \operatorname{Hom}_{\tau_{c}}\left(X, l^{\infty}(I)\right)
$$

is exact. Since for any object $X$ of $\mathcal{T} c$,

$$
\operatorname{Hom}_{\mathcal{T}_{c}}\left(X, l^{\infty}(I)\right) \simeq \operatorname{Hom}_{\mathcal{P r o}(\mathcal{B} a n)}\left(\mathrm{S}(X), “ l^{\infty}(I) "\right)
$$


the conclusion follows from Proposition 4.3.3.

Definition 4.3.10. $\quad$ Let

$$
\mathrm{L}: \operatorname{P} r o(\operatorname{Ban}) \rightarrow \mathcal{T} c
$$

be the composite functor

$$
\operatorname{Pro}(\operatorname{Ban}) \stackrel{\operatorname{Pro}(l)}{\longrightarrow} \operatorname{Pro}(\mathcal{T} c) \stackrel{\mathrm{L} \tau_{c}}{\longrightarrow} \mathcal{T} c
$$

where $I: \mathcal{B}$ an $\rightarrow \mathcal{T} c$ is the canonical embedding. In other words, for any functor $X: \mathcal{J}^{\text {op }} \rightarrow$ Ban, we set

$$
\mathrm{L}\left(“ \lim _{i \in \mathfrak{J}} ” X(i)\right)=\lim _{i \in \mathfrak{J}} X(i)
$$

where the projective limit is taken in $\mathcal{T} c$.

Proposition 4.3.11. For any object $E$ of $\mathcal{T} c$ and any object $X$ of Pro(Ban), we have the adjunction formula:

$$
\operatorname{Hom}_{\mathcal{I}_{c}}(E, \mathrm{~L}(X)) \simeq \operatorname{Hom}_{\mathcal{P}_{r o(\mathcal{B} a n)}}(\mathrm{S}(E), X) .
$$

In particular, L preserves projective limits and $S$ preserves inductive limits.

Proof. Let $E$ be an object of $\mathcal{T} c$ and let $X: \mathcal{J}^{\mathrm{op}} \rightarrow \mathcal{B}$ an be an object of $\operatorname{Pro}(\mathcal{B} a n)$. Then, we get successively

$$
\begin{aligned}
\operatorname{Hom}_{\mathcal{T}_{c}}(E, \mathrm{~L}(X)) & \simeq \lim _{i \in \mathcal{J}} \operatorname{Hom}_{\mathcal{T}_{c}}(E, X(i)) \\
& \simeq \lim _{i \in \mathcal{J}} \operatorname{Hom}_{\mathcal{P r o}(\mathcal{B} a n)}(\mathrm{S}(E), “ X(i) ") \\
& \simeq \operatorname{Hom}_{\mathcal{P}_{r o}(\mathcal{B} a n)}(\mathrm{S}(E), X) .
\end{aligned}
$$

Lemma 4.3.12. For any object $E$ of $\mathcal{T} c$ with $P$ as system of semi-norms, we have

$$
\mathrm{L}(\mathrm{S}(E)) \simeq \varliminf_{p \in P} \widehat{E}_{p} \simeq \widehat{E} .
$$

Proof. The first isomorphism follows from the definitions. As for the second one, we refer to [6, Chap. IV, \& 19, 9.(1)(p. 231)]. 
Proposition 4.3.13. The functor

$$
\mathrm{RCpl}: D^{+}(\mathcal{T} c) \rightarrow D^{+}(\mathcal{T} c)
$$

is canonically isomorphic to

$$
\mathrm{RL} \circ \mathrm{S} \text {. }
$$

Proof. By Lemma 4.3.12, $\mathrm{Cpl}=\mathrm{L} \circ \mathrm{S}$ and by Proposition 4.3.9, the functor $\mathrm{S}$ is exact, so we have to prove that

$$
R(\mathrm{~L} \circ \mathrm{S})=\mathrm{RL} \circ \mathrm{RS} .
$$

The objects of an injective resolution in $\mathcal{T} c$ may be assumed to be of the form $E \times \prod_{i \in I} F_{i}$, where $E$ is an object of $\tau c$ with the weakest topology and $F_{i}$ is an injective Banach space for any $i \in I$. We have

$$
\mathrm{S}\left(E \times \prod_{i \in I} F_{i}\right) \simeq \mathrm{S}(E) \times \prod_{i \in I} \mathrm{~S}\left(F_{i}\right) \simeq \prod_{i \in I} " F_{i} .
$$

Since by [9, Proposition 7.3.9], $\Pi_{i \in I}$ " $F_{i}$ " is L-acyclic, $\mathrm{S}\left(E \times \Pi_{i \in I} F_{i}\right)$ is also L-acyclic and the conclusion follows.

Corollary 4.3.14. For any object $E$ of $\mathcal{T} c$, we have

$$
\mathrm{RCpl}(E) \simeq \operatorname{RCpl}(\operatorname{Sep}(E)) \simeq \operatorname{RCpl}(\operatorname{Cpl}(E)) .
$$

Proof. This follows from Proposition 4.3.13 and Proposition 4.3.7 keeping in mind that $\operatorname{Cpl}(E) \simeq \operatorname{Cpl}(\operatorname{Sep}(E)$ ).

Corollary 4.3.15. Let $E$ be an object of $\mathcal{T} c$ with $P$ as system of semi-norms. Then, the following conditions are equivalent.

(i) E is cohomologically complete;

(ii) $\mathrm{RL} \circ \mathrm{S}(E) \simeq E$;

(iii) $\mathrm{R} \lim _{p \in P} \widehat{E}_{p} \simeq E$.

Proof. The equivalence between (i) and (ii) follows from Proposition 4.3.13. phisms

The fact that (ii) is equivalent to (iii) follows from the isomor- 


$$
\mathrm{RL} \circ \mathrm{S}(E) \simeq \mathrm{RL}\left(“ \lim _{p \in P} ” \widehat{E}_{p}\right) \simeq \mathrm{R} \lim _{p \in P} \widehat{E}_{p} \text {. }
$$

Theorem 4.3.16. The functor

$$
\mathrm{RL}: D^{+}(\mathcal{P} r o(\operatorname{Ban})) \rightarrow D_{c c}^{+}(\mathcal{T} c)
$$

is an equivalence of categories.

Proof. The functors $\mathrm{S}: \mathcal{T} c \rightarrow \operatorname{Pro}(\mathcal{B} a n)$ being exact, it gives rise to a functor

$$
\mathrm{S}: D_{c c}^{+}(\mathcal{T} c) \rightarrow D^{+}(\mathcal{P} r o(\operatorname{Ban})) .
$$

First, by Corollary 4.3 .15 , for any object $E^{\cdot}$ of $D_{c c}^{+}(\mathcal{T} c)$, we have

$$
\mathrm{RL} \circ \mathrm{S}\left(E^{\cdot}\right) \simeq E^{*}
$$

Next, consider a complex $X$

$$
0 \rightarrow X^{-k} \rightarrow X^{-k+1} \rightarrow \cdots
$$

of $D^{+}(\operatorname{Pro}(\mathcal{B} a n))$. We know that $X^{\cdot}$ has an injective resolution by objects of the type $\Pi_{i \in \jmath}$ " $I_{i}$ " where each $I_{i}$ is an injective object of $\mathcal{B} a n$. Since we have

$$
\left.\mathrm{S}\left(\mathrm{L} \prod_{i \in \mathcal{J}} " I_{i} "\right)\right) \simeq \mathrm{S}\left(\prod_{i \in \mathcal{J}} I_{i}\right) \simeq \prod_{i \in \mathcal{J}} \text { “I } I_{i}
$$

we get $\mathrm{S} \circ \mathrm{RL}\left(X^{*}\right) \simeq X^{*}$ in $D^{+}(\mathcal{P} r o(\mathcal{B} a n))$. Therefore, for any $X^{*} \in D^{+}$ ( $\operatorname{Pro}(\mathcal{B} a n))$, we have

$$
(\mathrm{RL} \circ \mathrm{S})\left(\mathrm{RL}\left(X^{*}\right)\right) \simeq \mathrm{RL}\left(\mathrm{S} \circ \mathrm{RL}\left(X^{*}\right)\right) \simeq \mathrm{RL}\left(X^{*}\right)
$$

and $\operatorname{RL}\left(X^{*}\right) \in D_{c c}^{+}\left(\mathcal{T}_{c}\right)$. The conclusion follows.

Corollary 4.3.17. Let $E$ and $F$ be two objects of $\mathcal{T} c$ and let $P$ and $Q$ be their respective systems of semi-norms. If $F$ is cohomologically complete, then

$$
\operatorname{RHom}_{\mathcal{T} c}(E, F) \simeq \mathrm{R} \underset{q \in Q}{\lim _{q \in P}} \underset{p \in \lim _{p}}{\mathrm{RHom}_{B a n}}\left(\widehat{E}_{p}, \widehat{F}_{q}\right) .
$$


Proof. By Proposition 2.2.14, we know that $E \simeq \mathrm{R} \lim _{p \in P} E_{p}$ and since $F$ is cohomologically complete, by Corollary $4.3 .15 F \simeq \mathrm{R} \lim _{q \in Q} \widehat{F}_{q}$. Then,
we have successively

$$
\begin{aligned}
& \mathrm{RHom}_{\mathcal{T}_{c}}(E, F) \simeq \mathrm{RHom}\left(\mathrm{R} \varliminf_{p \in P} E_{p}, \mathrm{R} \varliminf_{q \in Q} \widehat{F}_{q}\right) \\
& \simeq \mathrm{R} \varliminf_{q \in Q} \mathrm{RHom}\left(\mathrm{R} \varliminf_{p \in P} E_{p}, \hat{F}_{q}\right) \\
& \simeq \mathrm{R} \varliminf_{q \in Q} \lim _{p \in P} \operatorname{RHom}_{\tau_{c}}\left(E_{p}, \hat{F}_{q}\right) \\
& \simeq \mathrm{R} \varliminf_{q \in Q} \lim _{p \in P} \mathrm{RHom}_{\mathcal{B} a n}\left(\widehat{E}_{p}, \widehat{F}_{q}\right)
\end{aligned}
$$

where the isomorphism (*) follows from [9, Proposition 3.6.3] and the isomorphism (**) from Proposition 2.2.4.

\section{§ 4.4. Equivalence between $D^{+}(\mathcal{F} r)$ and $D^{+}\left(\mathcal{P} r o_{\mathbb{N}}(\mathcal{B} a n)\right)$}

Definition 4.4.1. The category of Fréchet spaces is the full additive subcategory of $\mathcal{T} c$ whose objects are the Fréchet spaces. We denote it by $\mathcal{F} r$.

Proposition 4.4.2. Let $f: E^{\prime} \rightarrow F$ be a morphism of $\mathcal{F} r$.

(i) The kernel of $f$ is the subspace $f^{-1}(0)$ of $E$ endowed with the induced topology.

(ii) The cokernel of $f$ is the quotient space $F / \overline{f(E)}$ endowed with the quotient topology.

(iii) The image of $f$ is the subspace $\overline{f(E)}$ of $F$ endowed with the induced topology.

(iv) The coimage of $f$ is the quotient space $E / f^{-1}(0)$ endowed with the quotient topology.

Corollary 4.4.3. Let $f: E \rightarrow F$ be a morphism of $\mathcal{F} r$. The following conditions are equivalent :

(i) f is strict in $\mathcal{F} r$, 
(ii) $f$ is relatively open,

(iii) $f(E)$ is closed.

In particular, a morphism of $\mathcal{F} r$ is strict if and only if it is strict as a morphism of $\mathcal{T} c$.

Proof. It is sufficient to work as for $\widehat{\mathcal{T}}_{c}$ keeping in mind the closed graph theorem.

Corollary 4.4.4. Let $f: E \rightarrow F$ be a morphism of $\mathcal{F} r$. Then, $f$ is a strict monomorphism (resp. epimorphism) of $\mathcal{F} r$ if and only if $f$ is a strict monomorphism (resp. epimorphism) of $\mathcal{T} c$.

Proposition 4.4.5. The category $\mathcal{F} r$ is quasi-abelian.

Proof. We know that $\mathcal{F} r$ is additive and that any morphism of $\mathcal{F} r$ has a kernel and a cokernel.

Consider the cartesian square

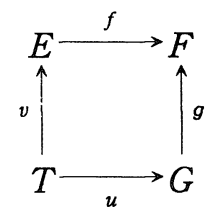

of $\mathcal{F} r$ where $f$ is a strict epimorphism. By Corollary 4.4.4, $f$ is a strict epimorphism of $\mathcal{T} c$ and since $\mathcal{T} c$ is quasi-abelian, it follows that $u$ is a strict epimorphism in $\mathcal{T} c$ and also in $\mathcal{F} r$.

Finally, consider the cocartesian square

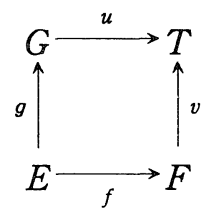

of $\mathcal{F} r$ where $f$ is a strict monomorphism. Denote $\alpha$ the morphism

$$
\left(\begin{array}{c}
g \\
-f
\end{array}\right): E \rightarrow G \oplus F .
$$

Recall that

$$
T \simeq \operatorname{coker}(\alpha) \simeq(G \oplus F) / \overline{\alpha(E)} .
$$


By Corollary 4.4.4, $f$ is a strict monomorphism in $\mathcal{T} c$. Then, $f$ is injective and for any semi-norm $p_{E}$ of $E$, there is a semi-norm $p_{F}$ of $F$ such that

$$
p_{E}(e) \leq C p_{F}(f(e)) \quad \forall e \in E
$$

for some $C>0$. It follows that $\alpha$ is injective and that

$$
p_{E}(e) \leq C \sup \left(p_{F}(-f(e)), p_{G}(g(e))\right) \leq C p_{G \oplus F}(\alpha(e))
$$

where $p_{G}$ is an arbitrary semi-norm of $G$ and $p_{G \oplus F}(x, y)=$ $\sup \left(p_{F}(x), p_{G}(y)\right)$. Hence, $\alpha$ is a strict monomorphism and its image is closed. Consequently,

$$
\text { coker } \alpha \simeq(G \oplus F) / \alpha(E)
$$

and the cokernel of $\alpha$ in $\mathcal{F} r$ coincides with the cokernel of $\alpha$ in $\mathcal{T} c$. Then, the square $\left(^{*}\right)$ is cocartesian in $\mathcal{T} c$. By Corollary 4.4.4, $f$ is a strict monomorphism of $\mathcal{T} c$ and since $\mathcal{T} c$ is quasi-abelian, it follows that $u$ is a strict monomorphism in $\mathcal{T} c$ and also in $\mathcal{F} r$.

Proposition 4.4.6. The category Fr has enough injective objects.

Proof. This follows from Proposition 2.1.12 using the fact that countable products of Banach spaces are Fréchet spaces.

Proposition 4.4.7. Fréchet spaces are cohomologically complete.

Proof. Let $F$ be a Fréchet space. By Proposition 4.4.6, $F$ has an injective resolution $I^{*}$ in $\mathcal{F} r$ such that $I^{k}$ is also an injective object of $\mathcal{T}$ c. Therefore,

$$
\operatorname{RCpl}(F) \simeq \operatorname{Cpl}\left(I^{*}\right) \simeq I^{*} \simeq F .
$$

Definition 4.4.8. A small category $\mathcal{J}$ is called countable if the set of objects of $\mathcal{J}$ is countable and if the set of morphisms between two arbitrary objects of $\mathcal{J}$ is also countable.

Definition 4.4.9. Let $\mathcal{C}$ be an arbitrary category. A countable pro-object of $\mathcal{C}$ is a functor $X: \mathcal{J}^{\text {op }} \rightarrow \mathcal{C}$ from some countable filtering category $\mathcal{J}$ to $\mathcal{C}$. The category of countable pro-objects of $\mathcal{C}$ is denoted by 


$$
\operatorname{Pro}_{\mathbb{N}}(\mathcal{C}) \text {. }
$$

Remark 4.4.10. Working as in [1], one can check easily that for any filtering countable category $\mathcal{J}$, there is a countable filtering ordered set $I$ and a cofinal functor

$$
\Phi: I \rightarrow J
$$

Thanks to $[10$, Lemma 5.1$]$, one may even assume that $I=\mathbb{N}$. In particular, for any countable pro-object $X$ of a category $\mathcal{C}$, we may find a functor

$$
X^{\prime}: \mathbb{N} \rightarrow \mathcal{C}
$$

such that

$$
X=“ \lim _{n \in \mathbb{N}} " X_{n}^{\prime} .
$$

Proposition 4.4.11. If $\mathcal{E}$ is a quasi-abelian category then $\operatorname{Pro}_{\mathbb{N}}(\mathcal{E})$ is a quasi-abelian category.

Proof. Work as in [9, Proposition 7.1.5]

Lemma 4.4.12. For any object $X: \mathcal{J}^{o p} \rightarrow \mathcal{B}$ an of $\operatorname{Pro}_{\mathbb{N}}(\mathcal{B} a n)$,

$$
\mathrm{L}(X)=\lim _{i \in J} X(i)
$$

is a Fréchet space. In particular, we may consider the functor

$$
\mathrm{L}: \mathcal{P r o}_{\mathbb{N}}(\mathcal{B} a n) \rightarrow \mathcal{F} r \text {. }
$$

Corollary 4.4.13. The functor

$$
\mathrm{L}: \mathcal{P r o}_{\mathbb{N}}(\mathcal{B} a n) \rightarrow \mathcal{F} r
$$

is right derivable and its derived functor

$$
\mathrm{RL}: D^{+}\left(\mathcal{P r o}_{\mathbb{N}}(\mathcal{B} a n)\right) \rightarrow D^{+}(\mathcal{F} r)
$$

is an equivalence of categories.

Proof. Since $\mathcal{B}$ an has enough injective objects, $\mathcal{P} r o_{\mathbb{N}}(\mathcal{B} a n)$ has 
enough injective objects and $\mathrm{L}: \mathcal{P}_{0_{\mathbb{N}}}(\mathcal{B} a n) \rightarrow \mathcal{F} r$ is right derivable. The functor $\mathrm{S}: \mathcal{F} r \rightarrow \mathcal{P}_{\text {ro }}(\mathcal{B} a n)$ being exact, it gives rise to a functor

$$
\mathrm{S}: D^{+}(\mathcal{F r}) \rightarrow D^{+}\left(\mathcal{P r o}_{\mathbb{N}}(\mathcal{B} a n)\right)
$$

Consider a complex $F$

$$
0 \rightarrow F^{-k} \rightarrow F^{-k+1} \rightarrow F^{-k+2} \rightarrow \cdots
$$

of $D^{+}(\mathcal{F} r)$. For any $n \geq-k$, since $F^{n}$ is cohomologically complete, we have

$$
\mathrm{RL} \circ \mathrm{S}\left(F^{n}\right) \simeq F^{n} \simeq \mathrm{L} \circ \mathrm{S}\left(F^{n}\right)
$$

where the last isomorphism follows from Lemma 4.3.12. Hence, $\mathrm{S}\left(F^{\circ}\right)$ is a L-acyclic resolution of $\mathrm{S}\left(F^{\circ}\right)$ and we have

$$
\mathrm{RL} \circ \mathrm{S}\left(F^{\cdot}\right) \simeq \mathrm{L} \circ \mathrm{S}\left(F^{*}\right) \simeq F^{*}
$$

Hence, the complexes of $D^{+}(\mathcal{F} r)$ are cohomologically complete. As in the proof of Theorem 4.3.16, one checks that the functor

$$
\mathrm{RL}: D^{+}\left(\mathcal{P r o}_{\mathbb{N}}(\mathcal{B} a n)\right) \rightarrow D^{+}(\mathcal{F} r)
$$

is an equivalence of categories and its quasi-inverse is given by

$$
\mathrm{S}: D^{+}(\mathcal{F} r) \rightarrow D^{+}\left(\mathcal{P r o}_{\mathbb{N}}(\mathcal{B} a n)\right)
$$

\section{$\S 5 . \quad$ Duality Functors}

\section{$\S 5.1$ The Inductive Dual}

Remark 5.1.1. Let us recall that if $E$ is a semi-normed space, then the dual of $E$, denoted here by $\mathrm{D}(E)$, is a Banach space. If $p$ is the semi-norm of $E$, then the norm of $\mathrm{D}(E)$ is defined by

$$
\|\tau\|_{D(E)}=\sup _{p(x) \leq 1}|\tau(x)|
$$

for any $\tau \in \mathrm{D}(E)$.

Recall that if $E$ is a semi-normed space, we have the isomorphism of 
Banach spaces $\mathrm{D}(E) \simeq \mathrm{D}(\widehat{E})$. In particular, if $E$ is an object of $\mathcal{T}_{c}$ with the weakest topology, then $\mathrm{D}(E) \simeq 0$.

Recall also that the duality functor

$$
\mathrm{D}: \mathcal{B} a n \rightarrow(\mathcal{B} a n)^{\mathrm{op}}
$$

is exact. As a matter of fact, consider a strictly exact sequence

$$
0 \rightarrow E \stackrel{e}{\rightarrow} F \stackrel{f}{\rightarrow} G \rightarrow 0
$$

of Banach spaces. Since $\mathbb{C}=l^{\infty}(\{0\})$ is an injective object of $\mathcal{B} a$, the sequence

$$
0 \rightarrow \mathrm{D}(G) \stackrel{\mathrm{D}(f)}{\longrightarrow} \mathrm{D}(F) \stackrel{\mathrm{D}(e)}{\longrightarrow} \mathrm{D}(E) \rightarrow 0
$$

is an exact sequence of vector spaces. Since the images of $\mathrm{D}(f)$ and $\mathrm{D}(\boldsymbol{e})$ are closed, the Banach homomorphism theorem shows that $\mathrm{D}(f)$ and $\mathrm{D}(e)$ are strict.

Let $X$ be an object of $\mathcal{T} c$. Recall that a set of continuous linear functionals

$$
E=\left\{f_{i}: i \in I, f_{i}: X \rightarrow \mathbb{C}\right\}
$$

is equicontinuous if for any $\epsilon>0$, there is an absolutely convex neighborhood of zero $V$ in $X$ such that

$$
\left|f_{i}(v)\right| \leq \epsilon \quad \forall v \in V, \quad \forall i \in I .
$$

It is equivalent to ask that there is an absolutely convex neighborhood of zero $V$ in $X$ such that $E \subset V^{\circ}$ or that $E^{\circ}$ is a neighborhood of zero in $X$. In particular, the polar of any semi-ball of $X$ is equicontinuous.

Definition 5.1.2. We denote by

$$
\mathrm{D}_{\mathrm{i}}: \mathcal{T} c \rightarrow \mathcal{T} c^{\mathrm{op}}
$$

the inductive dual functor, which associates to any object $X$ of $\mathcal{T}_{c}$ the dual space $X^{\prime}$ endowed with the inductive topology. In this topology, a basis of neighborhoods of zero is formed by the absolutely convex subsets of $X^{\prime}$ which absorb any equicontinuous set. 
Lemma 5.1.3. If $X$ is a semi-normed space, then $\mathrm{D}_{\mathrm{i}}(X) \simeq D(X)$.

Proof. Recall that if $p$ is the semi-norm of $X$, then a basis of neighborhoods of zero in $\mathrm{D}(X)$ is given by $\left\{b_{p}(r)^{\circ}: r>0\right\}$. The conclusion follows easily.

Proposition 5.1.4. Let $E$ be an object of $\mathcal{T} c$ and let $P$ be its system of semi-norms. Then, we have

$$
\mathrm{D}_{\mathrm{i}}(E) \simeq \lim _{p \in P} \mathrm{D}\left(E_{p}\right) \simeq \lim _{p \in P} \mathrm{D}\left(\widehat{E}_{p}\right) .
$$

Proof. By Lemma 5.1.3, it is sufficient to show that $\lim _{p \in P} D_{i}\left(E_{p}\right) \simeq$ $\mathrm{D}_{\mathrm{i}}(E)$. Consider the continuous linear map

$$
u: \lim _{p \in P} \mathrm{D}_{\mathrm{i}}\left(E_{p}\right) \rightarrow \mathrm{D}_{\mathrm{i}}(E)
$$

defined by

$$
u \circ r_{p}=\mathrm{D}_{\mathrm{i}}\left(e_{p}\right) \quad \forall p \in P
$$

where $e_{p}: E \rightarrow E_{p}$ is the continuous identity map. This map $u$ is clearly bijective.

To conclude, it is sufficient to show that $u^{-1}$ is continuous. Consider a subset $U$ of $D_{i}(E)$ such that $u^{-1}(U)$ is a neighborhood of zero in $\lim _{p \in P} D_{i}\left(E_{p}\right)$. Let us show that $U$ is a neighborhood of zero in $\mathrm{D}_{\mathrm{i}}(E)$.

Consider a semi-ball $b_{p}(1)$ of $E$. Denote $\left(b_{p}(1)\right)_{E^{\prime}}^{\circ}\left(\right.$ resp. $\left.\left(b_{p}(1)\right)_{E_{p}^{\prime}}^{\circ}\right)$ the polar of $b_{p}(1)$ in $E^{\prime}$ (resp. $E_{p}^{\prime}$ ). Since

$$
r_{p}^{-1}\left(u^{-1}(U)\right)=\left(u \circ r_{p}\right)^{-1}(U)=\left(D_{\mathrm{i}}\left(e_{p}\right)\right)^{-1}(U)
$$

is a neighborhood of zero in $D_{\mathrm{i}}\left(E_{p}\right),\left(D_{\mathrm{i}}\left(e_{p}\right)\right)^{-1}(U)$ absorbs $\left(b_{p}(1)\right)_{E_{p}^{\prime}}^{0}$ Hence, there is $C>0$ such that

$$
\left(b_{p}(1)\right)_{E_{p}^{\prime}}^{\supset} \subset C\left(D_{\mathrm{i}}\left(e_{p}\right)\right)^{-1}(U) .
$$

It follows that

$$
\left(b_{p}(1)\right)_{E^{\prime}}^{\circ}=\left(D_{\mathrm{i}}\left(e_{p}\right)\right)\left(\left(b_{p}(1)\right)_{E_{p}^{\prime}}^{\circ}\right) \subset C\left(D_{\mathrm{i}}\left(e_{p}\right)\right)\left[\left(D_{\mathrm{i}}\left(e_{p}\right)\right)^{-1}(U)\right] \subset C U .
$$

Therefore, $U$ absorbs the polar of any semi-ball of $E$ and $U$ is a neighborhood of zero in $\mathrm{D}_{\mathrm{i}}(E)$. 
The last isomorphism follows from Remark 5.1.1.

Proposition 5.1.5. For any family $\left(X_{\alpha}\right)_{\alpha \in A}$ of objects of $\mathcal{T} c$, we have

$$
\mathrm{D}_{\mathrm{i}}\left(\prod_{\alpha \in A} X_{\alpha}\right) \simeq \bigoplus_{\alpha \in A} \mathrm{D}_{\mathrm{i}}\left(X_{\alpha}\right) \text {. }
$$

Proof. Denote

$$
f: \bigoplus_{\alpha \in A} \mathrm{D}_{\mathrm{i}}\left(X_{\alpha}\right) \rightarrow \mathrm{D}_{\mathrm{i}}\left(\prod_{\alpha \in A} X_{\alpha}\right)
$$

the canonical morphism of $\mathcal{T} c$ induced by the morphisms

$$
\mathrm{D}_{\mathrm{i}}\left(p_{\alpha}\right): \mathrm{D}_{\mathrm{i}}\left(X_{\alpha}\right) \rightarrow \mathrm{D}_{\mathrm{i}}\left(\prod_{\alpha \in A} X_{\alpha}\right)
$$

where

$$
p_{\alpha}: \prod_{\alpha \in A} X_{\alpha} \rightarrow X_{\alpha}
$$

is the canonical projection. It is well-known (see e.g. [6, Chap. IV, \& 22 , 5.(2) (p. 284)] that $f$ is a bijection. Hence, it is sufficient to prove that it is open.

Consider a closed neighborhood of zero $U$ in $\oplus_{\alpha \in A} \mathrm{D}_{\mathrm{i}}\left(X_{\alpha}\right)$. Then,

$$
U \supset \overline{\left\langle\bigcup_{\alpha \in A} \sigma_{\alpha}\left(U_{\alpha}\right)\right\rangle}
$$

where each $U_{\alpha}$ is a closed absolutely convex neighborhood of zero in $\mathrm{D}_{\mathrm{i}}\left(X_{\alpha}\right)$ and $\sigma_{\alpha}: \mathrm{D}_{\mathrm{i}}\left(X_{\alpha}\right) \rightarrow \oplus_{\alpha \in A} \mathrm{D}_{\mathrm{i}}\left(X_{\alpha}\right)$ is the canonical embedding. Consider an equicontinuous set $E$ of $\left(\prod_{\alpha \in A} X_{\alpha}\right)^{\prime}$. There is an absolutely convex neighborhood of zero $V$ in $\Pi_{\alpha \in A} X_{\alpha}$ such that

$$
E \subset V^{\circ} \text {. }
$$

We may assume that

$$
V=\prod_{\alpha \in A} V_{\alpha}
$$

where each $V_{\alpha}$ is a closed absolutely convex neighborhood of zero in $X_{\alpha}$ and the set

$$
\left\{\alpha \in A: V_{\alpha} \neq X_{\alpha}\right\}
$$


is finite. Since $U_{\alpha}$ is a neighborhood of zero in $\mathrm{D}_{\mathrm{i}}\left(X_{\alpha}\right)$, there is $C_{\alpha}>0$ such that

$$
V_{\alpha}^{\circ} \subset C_{\alpha} U_{\alpha}
$$

If $V_{\alpha}=X_{\alpha}$, then $V_{\alpha}^{\circ}=X_{\alpha}^{\circ}=0$ and we may assume $C_{\alpha}=0$. Then, setting $C=\sup _{\alpha \in A} C_{\alpha}$, we get

$$
E \subset\left(\prod_{\alpha \in A} V_{\alpha}\right)^{\circ} \subset f\left(\overline{\left\langle\bigcup_{\alpha \in A} \sigma_{\alpha}\left(V_{\alpha}^{\circ}\right)\right\rangle}\right) \subset C f\left(\overline{\left\langle\bigcup_{\alpha \in A} \sigma_{\alpha}\left(U_{\alpha}\right)\right\rangle}\right) \subset C f(U)
$$

where the second inclusion follows from e. g. [6, Chap. IV, § 22, 5.(1)(p. 283)]. Hence, $f(U)$ is a neighborhood of zero in $\mathrm{D}_{\mathrm{i}}\left(\Pi_{\alpha \in A} X_{\alpha}\right)$.

Proposition 5.1.6. The functor $\mathrm{D}_{\mathrm{i}}: \mathcal{T} c \rightarrow \mathcal{T} c^{\mathrm{op}}$ is left exact.

Proof. Consider a strictly exact sequence

$$
0 \rightarrow X \stackrel{f}{\rightarrow} Y \stackrel{g}{\rightarrow} Z \rightarrow 0
$$

of $\mathcal{T} c$. We know that the sequence

$$
\mathrm{D}_{\mathrm{i}}(Z) \stackrel{\mathrm{D}_{\mathrm{i}}(g)}{\longrightarrow} \mathrm{D}_{\mathrm{i}}(Y) \stackrel{\mathrm{D}_{\mathrm{i}}(f)}{\longrightarrow} \mathrm{D}_{\mathrm{i}}(X) \rightarrow 0
$$

is algebraically exact and that the maps $\mathrm{D}_{\mathrm{i}}(g)$ and $\mathrm{D}_{\mathrm{i}}(f)$ are continuous. Therefore, it is sufficient to show that $\mathrm{D}_{\mathrm{i}}(f)$ is strict, i.e. $\mathrm{D}_{\mathrm{i}}(f)$ is relatively open.

Let $V$ be a neighborhood of zero in $D_{\mathrm{i}}(Y)$ and $E$ be an equicontinuous subset of $X^{\prime}$. We have to show that $\mathrm{D}_{\mathrm{i}}(f)(V)$ absorbs $E$. Let $p_{X}$ be a continuous semi-norm of $X$ such that $E \subset b_{p_{X}}(1)^{\circ}$. Since $f$ is a strict monomorphism, there is a continuous semi-norm $p_{Y}$ on $Y$ such that

$$
p_{X}(x) \leq p_{Y}(f(x)) \quad \forall x \in X .
$$

Let $\tau_{X} \in b_{p_{X}}(1)^{\circ}$. We have $\left|\tau_{X}(x)\right| \leq p_{X}(x)$ and the Hahn-Banach theorem shows that there is $\tau_{Y} \in Y^{\prime}$ such that

$$
\left|\tau_{Y}(y)\right| \leq p_{Y}(y) \quad \text { and } \quad \tau_{Y} \circ f=\tau_{X} .
$$

It follows that $b_{p_{X}}(1)^{\circ} \subset D_{\mathrm{i}}(f)\left(b_{p_{Y}}(1)^{\circ}\right)$. Since $b_{p_{Y}}(1)^{\circ}$ is an equicontinuous subset of $Y^{\prime}$, there is $C>0$ such that $b_{p_{Y}}(1)^{\circ} \subset C V$. It follows that 


$$
b_{p_{X}}(1)^{\circ} \subset \mathrm{D}_{\mathrm{i}}(f)\left(b_{p_{Y}}(1)^{\circ}\right) \subset C \mathrm{D}_{\mathrm{i}}(f)(V) .
$$

Hence, the conclusion.

Remark 5.1.7. Let $\mathcal{J}$ be a small category and let $\mathcal{E}$ be an additive category with exact products. Recall that in [9], we defined a functor

$$
\Pi: \mathcal{E}^{\mathrm{ob}(\jmath)} \rightarrow \mathcal{E}^{\mathrm{J}^{\mathrm{op}}}
$$

such that

and we established that

$$
\Pi(S)(i)=\prod_{\substack{\alpha \\ j \rightarrow i}} S(j)
$$

(a) for any object $E$ of $\varepsilon^{\text {op }}$, there is a strict monomorphism of the form $E \rightarrow \Pi(S)$ with $S$ in $\varepsilon^{\mathrm{ob}(\jmath)}$,

(b) any object of the form $\Pi(S)$ with $S$ in $\mathcal{E}^{\mathrm{Ob(J)}}$ is $\varliminf_{i \in \mathcal{J}}$-acyclic,

(c) if $S$ is an object of $\mathcal{E}^{\mathrm{Ob}(\mathcal{J})}$ with $S(i)$ injective for any $i \in \mathcal{J}$, then $\Pi(S)$ is injective in $\mathcal{E}^{\mathcal{J}^{\mathrm{op}}}$.

We also established dual results when $\Pi$ is replaced by its dual counterpart $\amalg$.

Proposition 5.1.8. Let $\mathcal{J}$ be a small category. For any object $X$ of $\mathcal{T} c^{\mathrm{J}^{\mathrm{op}}}$, we have

$$
\mathrm{RD}_{\mathrm{i}}\left(\mathrm{R} \lim _{i \in \mathfrak{J}} X(i)\right) \simeq \mathrm{L} \lim _{i \in \mathfrak{J}}\left(\mathrm{RD}_{\mathrm{i}}(X)\right)(i) .
$$

Proof. We know that $X$ has an injective resolution in $\mathcal{T}_{c}{ }^{\text {op }}$ of the form

$$
0 \rightarrow \Pi\left(S^{0}\right) \rightarrow \Pi\left(S^{1}\right) \rightarrow \cdots
$$

where for $l \geq 0, S^{1}$ is an injective object of $\mathcal{T} c^{\mathrm{ob}(\jmath)}$.

On one hand, since for $l \geq 0$,

$$
\lim _{i \in \mathcal{J}} \Pi\left(S^{l}\right)(i) \simeq \prod_{i \in \mathcal{J}} S^{l}(i) \quad \text { and } \quad \mathrm{D}_{\mathrm{i}}\left(\prod_{i \in \mathcal{J}} S^{l}(i)\right) \simeq \bigoplus_{i \in \mathcal{J}} \mathrm{D}_{\mathrm{i}}\left(S^{l}(i)\right)
$$

and since a product of injective objects is an injective object, 
$\mathrm{RD}_{\mathrm{i}}(\mathrm{R} \underset{i \in \mathcal{J}}{\lim } X(i))$ is given by the complex

$$
\cdots \rightarrow \bigoplus_{i \in J} \mathrm{D}_{\mathrm{i}}\left(S^{1}(i)\right) \rightarrow \bigoplus_{i \in \mathrm{J}} \mathrm{D}_{\mathrm{i}}\left(S^{0}(i)\right) \rightarrow 0 .
$$

On the other hand, by Proposition 5.1.5, one can check easily that for $l \geq 0$

$$
\mathrm{D}_{\mathrm{i}}\left(\Pi\left(S^{l}\right)\right) \simeq \amalg\left(\mathrm{D}_{\mathrm{i}}\left(S^{l}\right)\right) .
$$

Then, $\mathrm{L} \underset{i \in \mathrm{lim}}{\lim }\left(\mathrm{RD}_{\mathrm{i}}(X)\right)(i)$ is given by the complex

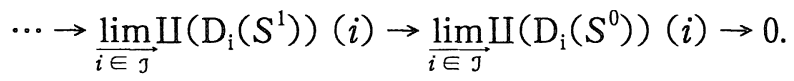

Since for $l \geq 0$, we have

$$
\underset{i \in \mathcal{J}}{\lim } \amalg\left(D_{\mathrm{i}}\left(S^{l}\right)\right)(i) \simeq \bigoplus_{i \in \mathrm{J}} \mathrm{D}_{\mathrm{i}}\left(S^{l}\right)(i) \simeq \bigoplus_{i \in \mathrm{J}} \mathrm{D}_{\mathrm{i}}\left(S^{l}(i)\right),
$$

the conclusion follows.

Proposition 5.1.9. If $X$ is a semi-normed space, then $\mathrm{RD}_{\mathrm{i}}(X) \simeq$ $\mathrm{D}(X)$.

Proof. We know that $X$ has an injective resolution of the form

$$
0 \rightarrow I^{0} \rightarrow I^{1} \rightarrow \cdots
$$

such that for $l \geq 0, I^{l}=E^{l} \times F^{l}$ where $E^{l}$ is an object of $\mathcal{T} c$ with the weakest topology and $F^{l}$ is an injective Banach space. Since for $l \geq 0, I^{l}$ is semi-normed, we have $\mathrm{D}_{\mathrm{i}}\left(I^{l}\right) \simeq \mathrm{D}\left(I^{l}\right)$. Moreover, since $\mathbb{C}=l^{\infty}(\{0\})$ is an injective object of $\mathcal{T} c$, the complex

$$
\cdots \rightarrow \mathrm{D}\left(I^{1}\right) \rightarrow \mathrm{D}\left(I^{0}\right) \rightarrow \mathrm{D}(X) \rightarrow 0
$$

is algebraically exact. Then, the image of any morphism of this complex is closed and by the Banach homomorphism theorem, the complex is strictly exact. Therefore,

$$
\mathrm{RD}_{\mathrm{i}}(X) \simeq \mathrm{D}(X) .
$$


Corollary 5.1.10. If $\left(X_{\alpha}\right)_{\alpha \in A}$ is a family of semi-normed spaces, then

$$
\mathrm{RD}_{\mathrm{i}}\left(\prod_{\alpha \in A} X_{\alpha}\right) \simeq \mathrm{D}_{\mathrm{i}}\left(\prod_{\alpha \in A} X_{\alpha}\right) \text {. }
$$

Proof. Since direct products and direct sums are exact in $\mathcal{T} c$, we may apply Proposition 5.1.8 and we have

$$
\mathrm{RD}_{\mathrm{i}}\left(\prod_{\alpha \in A} X_{\alpha}\right) \simeq \bigoplus_{\alpha \in A} \mathrm{RD}_{\mathrm{i}}\left(X_{\alpha}\right) .
$$

The conclusion follows from Proposition 5.1.9 and Proposition 5.1.5.

Proposition 5.1.11. For any object $X^{\cdot}$ of $D^{+}\left(\mathcal{T}_{c}\right)$, we have

$$
\operatorname{RD}_{\mathrm{i}}\left(\operatorname{RCpl}\left(X^{*}\right)\right) \simeq \mathrm{RD}_{\mathrm{i}}\left(X^{*}\right) .
$$

Hence,

$$
\mathrm{RD}_{\mathrm{i}}(E) \simeq \mathrm{RD}_{\mathrm{i}}(\operatorname{Sep}(E)) \simeq \mathrm{RD}_{\mathrm{i}}(\operatorname{Cpl}(E))
$$

for any object $E$ of $\mathcal{T} c$.

Proof. Consider an object $X^{*}$ of $D^{+}(\mathcal{T} c)$. We know that $X^{*}$ has an injective resolution by objects of the type

$$
I=E \times \prod_{\alpha \in A} F^{\alpha}
$$

where $E$ is an object of $\mathcal{T} c$ with the weakest topology and $F^{\alpha}$ is an injective Banach space. For such an object, we have

$$
\mathrm{Cpl}(I) \simeq \prod_{\alpha \in A} F^{\alpha} \quad \text { and } \quad \mathrm{D}_{\mathrm{i}}(I) \simeq \mathrm{D}_{\mathrm{i}}\left(\prod_{\alpha \in A} F^{\alpha}\right) .
$$

Since a product of injective objects is injective, we get

$$
\operatorname{RD}_{\mathrm{i}}(\operatorname{RCpl}(I)) \simeq \operatorname{RD}_{\mathrm{i}}(I) .
$$

Remark 5.1.12. Recall that since the functor D: $\operatorname{Ban} \rightarrow(\operatorname{Ban})^{\mathrm{op}}$ is exact, it induces an exact functor

$$
\operatorname{Pro}(\mathrm{D}): \operatorname{Pro}(\mathcal{B} a n) \rightarrow \operatorname{Pro}\left(\mathcal{B} a n^{\mathrm{op}}\right) \simeq(\mathcal{J} n d(\mathcal{B} a n))^{\mathrm{op}} \text {. }
$$


For any small filtering category $\mathcal{J}$ and any functor $X: \mathcal{J}^{\text {op }} \rightarrow \mathcal{B} a$, we have

$$
\operatorname{Pro}(\mathrm{D})\left(“ \lim _{i \in \mathfrak{J}} \text { "X(i)) = “ } \lim _{i \in \mathfrak{J}} \text { "D }(X(i))\right. \text {. }
$$

Proposition 5.1.13. The diagram

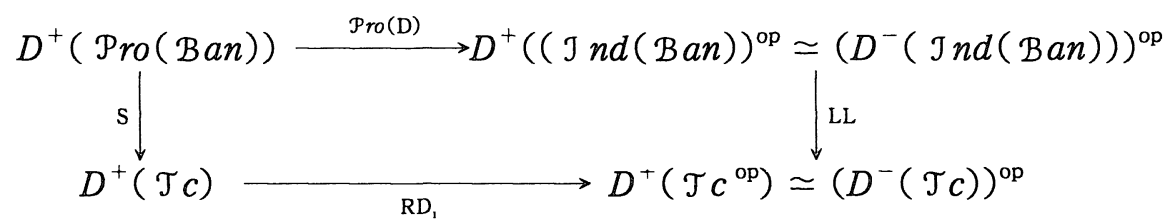

is commutative.

Proof. Consider an object $X^{\cdot}$ of $D^{+}(\mathcal{T} c)$. We know that $X^{\cdot}$ has an injective resolution by objects of the type

$$
I=E \times \prod_{\alpha \in A} F^{\alpha}
$$

where $E$ is an object of $\mathcal{T} c$ with the weakest topology and $F^{\alpha}$ is an injective Banach space. On one hand, we have

$$
\mathrm{D}_{\mathrm{i}}(I) \simeq \mathrm{D}_{\mathrm{i}}(E) \oplus \bigoplus_{\alpha \in A} \mathrm{D}_{\mathrm{i}}\left(F^{\alpha}\right) \simeq \mathrm{D}(E) \oplus \bigoplus_{\alpha \in A} \mathrm{D}\left(F^{\alpha}\right) \simeq \bigoplus_{\alpha \in A} \mathrm{D}\left(F^{\alpha}\right) .
$$

On the other hand, we have

$$
\mathrm{S}(I) \simeq \mathrm{S}(E) \times \prod_{\alpha \in A} \mathrm{~S}\left(F^{\alpha}\right) \simeq \prod_{\alpha \in A} " F^{\alpha ”} .
$$

Therefore, we get successively

$$
\begin{aligned}
& \operatorname{Pro}(\mathrm{D})(\mathrm{S}(I)) \simeq \operatorname{Pro}(\mathrm{D})\left(\prod_{\alpha \in A} " F^{\alpha "}\right) \simeq \operatorname{Pro}(\mathrm{D})\left(\lim _{J \in \mathcal{\mathcal { P }}_{f}(A)} \prod_{j \in J} “ F^{j "}\right) \\
& \simeq \lim _{J \in \widehat{\mathcal{P}_{f}}(A)} " \mathrm{D}\left(\prod_{j \in J} F^{j}\right) \\
& \simeq \lim _{J \in \overline{\mathcal{P}_{f}}(A)} " \bigoplus_{j \in J} \mathrm{D}\left(F^{i}\right) \simeq \bigoplus_{\alpha \in A} \text { “D }\left(F^{\alpha}\right) ” .
\end{aligned}
$$

Since

$$
\bigoplus_{\alpha \in A} \text { “D }\left(F^{\alpha}\right) ”
$$

is L-acyclic (see [9, Proposition 7.3.9]) and since 


$$
\mathrm{L}\left(\bigoplus_{\alpha \in A} \text { "D }\left(F^{\alpha}\right) "\right) \simeq \bigoplus_{\alpha \in A} \mathrm{~L}\left(" \mathrm{D}\left(F^{\alpha}\right) "\right) \simeq \bigoplus_{\alpha \in A} \mathrm{D}\left(F^{\alpha}\right),
$$

the conclusion follows.

Corollary 5.1.14. The diagram

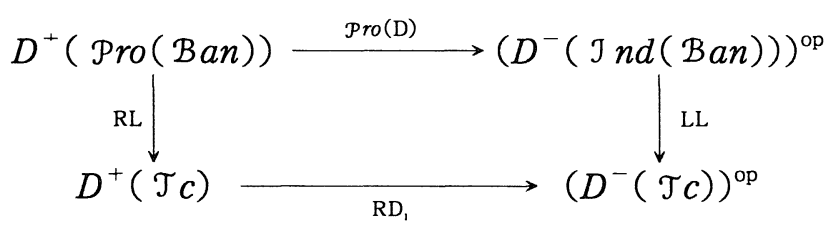

is commutative.

Proof. This follows directly from Theorem 4.3.16 and Proposition 5.1.13.

\section{§5.2. Relations with the Strong Dual}

Definition 5.2.1. We denote by

$$
\mathrm{D}_{\mathrm{b}}: \mathcal{T} c \rightarrow \mathcal{T} c^{\mathrm{op}}
$$

the strong dual functor which associates to any object $X$ of $\mathcal{T}_{C}$ the dual space $X^{\prime}$ endowed with the strong topology. In this topology, a basis of neighborhoods of zero is formed by the polar of the bounded subsets of $X$. The system of semi-norms is thus given by

$$
\left\{p_{B}: B \text { bounded subset of } X\right\}
$$

where $p_{B}$ is defined by

$$
p_{B}(\tau)=\sup _{x \in B}|\tau(x)| \quad \forall \tau \in \mathrm{D}_{\mathrm{b}}(X) .
$$

Proposition 5.2.2. The inductive topology is stronger than the strong topology.

Proof. Consider a bounded subset $B$ of an object $X$ of $\mathcal{T} c$ and let $E$ be an equicontinuous set. There is an absolutely convex neighborhood of zero $V$ in $X$ such that $E \subset V^{\circ}$. Since $B$ is bounded, there is $C>0$ such that $B \subset C V$. It follows that $E \subset C B^{\circ}$ and that $B^{\circ}$ is a neighborhood of 
zero in $\mathrm{D}_{\mathrm{i}}(X)$.

Proposition 5.2.3. If $X$ is a semi-normed space, then $\mathrm{D}(\mathrm{X}) \simeq$ $\mathrm{D}_{\mathrm{b}}(X)$.

Proof. This is clear since semi-balls are bounded in X.

Proposition 5.2.4. The functors

$$
\mathrm{RD}_{\mathrm{i}}: D^{+}\left(\mathcal{T}_{C}\right) \rightarrow D^{-}\left(\mathcal{T}_{C}\right)^{\mathrm{op}}
$$

and

$$
\mathrm{RD}_{\mathrm{b}}: D^{+}(\mathcal{T} c) \rightarrow D^{-}(\mathcal{T} c)^{\mathrm{op}},
$$

are canonically isomorphic.

Proof. Let $X$ be an object of $D^{+}(\mathcal{T} c)$. We know that $X$ has an injective resolution by objects of the type $I=E \times \Pi_{\alpha \in A} F^{\alpha}$ where $E$ is an object of $\mathcal{T} c$ with the weakest topology and $F^{\alpha}$ is an injective Banach space for any $\alpha \in A$. We have

$$
\mathrm{D}_{\mathrm{i}}(I) \simeq \mathrm{D}_{\mathrm{i}}(E) \oplus \bigoplus_{\alpha \in A} \mathrm{D}_{\mathrm{i}}\left(F^{\alpha}\right) \simeq \bigoplus_{\alpha \in A} \mathrm{D}\left(F^{\alpha}\right) .
$$

Moreover,

$$
\mathrm{D}_{\mathrm{b}}(I) \simeq \mathrm{D}_{\mathrm{b}}(E) \oplus \bigoplus_{\alpha \in A} \mathrm{D}_{\mathrm{b}}\left(F^{\alpha}\right) \simeq \bigoplus_{\alpha \in A} \mathrm{D}\left(F^{\alpha}\right)
$$

where the first isomorphism follows from [6, Chap. IV, \& 22, 5.(4)(p. 287)]. Therefore, $\mathrm{RD}_{\mathrm{i}}(X) \simeq \mathrm{RD}_{\mathrm{b}}(X)$.

\section{References}

[1] Artin, M., Grothendieck, A. and Verdier, J.-L. (eds.), Séminaire de géométrie algébrique du Bois-Marie 1963/64 (SGA4). Théorie des topos et cohomologie étale des schémas, 1, Lecture Notes in Math., no. 269, Springer, Berlin, 1972.

[2] Artin, M. and Mazur, B., Etale homotopy, Lecture Notes in Math., no. 100, Springer, Berlin, 1969.

[3] Bourbaki, N., Topologie générale (Chapitres 1 à 4), Éléments de Mathématiques, Diffusion C. C. L. S., Paris, 1971.

[4] Geiler, V. A., The projective objects in the category of locally convex spaces (Russian), Funkcional. Anal. i Priložen., 6 (1972), 79-80. 
[5] Kelley, J.-L. and Namioka, I., Linear topological spaces, Van Nostrand, Princeton, 1963.

[6] Köthe, G., Topological vector spaces I, Grundlehren der mathematischen Wissenschaften, no. 159, Springer, Berlin, 1969.

[7] Palamodov, V. P., Homological methods in the theory of locally convex spaces (Russian), Uspehi Mat. Nauk, 26 (1971), 3-65. For a translation see Russian Math. Surveys, 26 (1971), 1-64.

[8] Prosmans, F., Algèbre homologique quasi-abélienne, Mémoire de DEA, Université Paris 13, Villetaneuse (France), June 1995. Available on the web at 〈http: // www-math. univ-paris13. fr/ ${ }^{\sim}$ prosmans $\left./\right\rangle$.

[9] Derived limits in quasi-abelian categories, Bull. Soc. Roy. Sci. Liège, 68 (1999), 335-401.

[10] Derived projective limits of topological abelian groups, J. Funct. Anal., 162 (1999), 135-177.

[11] Schneiders, J.-P., Quasi-abelian categories and sheaves, Mém. Soc. Math. France (N. S.), 76 (1999). 
